\title{
Entrepreneurship, Innovation and Creativity: The Creative Process of Entrepreneurs and Innovators
}

\author{
Brian BARNARD $^{1 *}$ and Derrick HERBST ${ }^{2}$ \\ ${ }^{1}$ WITS Business School, The University of the Witwatersrand, South Africa \\ ${ }^{2}$ Henley Business School South Africa, University of Reading, Johannesburg, South Africa
}

\begin{abstract}
The study examines the creative process of entrepreneurs and innovators. It considers how several types of thinking - analytical, analogical, imaginary, intuitive - are involved in creativity and the creative process. It further considers how learning and composite thinking - the integration of the different types of thinking - are incorporated in the creative process. The subsequent analysis covers a number of aspects of creativity and the creative process: 1) attributes, 2) traits, 3) skill, 4) stimulants, 5) process, 6) method and technique, 7) imagination, 8) intuition and the sub-conscious, 9) problem statement, 10) referencing past solutions, 11) the solution space, 12) teams, and 13) factors of success in the market. The prominent role of the intuition and the sub-conscious in creativity is clear. Both the left-brain and rightbrain typically contribute to creativity. Perspective formation forms a key component of creativity. There is a semi-formal process to creativity. Both intuition and creativity itself can be developed. Given the array of factors that influence success in the market, it may be questioned whether creativity is essentially a prominent factor of entrepreneurship. Pure or raw creativity by itself is certainly not a sufficient factor of entrepreneurship but must first be combined with general business sense or acumen to guarantee innovation success.
\end{abstract}

Keywords: entrepreneurship, innovation, creativity, creative thinking, analytical thinking, analogical thinking, imaginary thinking, intuitive thinking, intuition, subconscious, learning, innovativeness, left-brain, right-brain.

JEL Classifications: O30, L26

\section{Introduction}

The study further examines the creative process of entrepreneurs and innovators. It investigates how different types of thinking - analytical, analogical, imaginary, intuitive - are involved in the creative process. It also considers how learning and composite thinking - the integration of the different types of types of

\footnotetext{
* Corresponding Author:

Brian Barnard, Wits Business School, University of the Witwatersrand, South Africa

Article History:

Received 3 April 2019| Accepted 24 June 2019 | Available Online 30 July 2019

Cite Reference:

Barnard, B. and Herbst, D., 2019. Entrepreneurship, Innovation and Creativity: The Creative Process of Entrepreneurs and Innovators. Expert Journal of Business and Management, 7(1), pp.107-146.

This paper has previously been included in an open access repository - SSRN.
} 
thinking - are involved in the creative process. One of the objectives is to note whether there is truly a leftbrain, right-brain divide when it comes to creativity - whether one side of the brain truly dominates during creativity, and in contributing to creativity and the creative process. Another objective is to consider how different types of thinking contribute to creativity and the creative process, and how they are combined during the creative process. Furthermore, it is considered whether and how learning takes place during the creative process. The study also follows a qualitative methodology by interviewing entrepreneurs and innovators, in the view that this too provides a unique perspective.

\section{Literature Review}

McFadzean (1998a) notes that creativity techniques can be classified into two groups: analytical and intuitive. Analytically-oriented techniques use a structure to generate a logical pattern of thought. These techniques include Force-Field Analysis and Progressive Abstraction. Intuitive techniques, on the other hand, allow the participant to make giant leaps or to observe images or symbols in order to arrive at a solution. Wishful Thinking and Metaphors are two techniques that can be classified under the intuitive category. Classical Brainstorming does not produce very many ideas that challenge or break away from a prevailing paradigm - this technique produces more paradigm preserving ideas than paradigm breaking ideas. This is because these techniques only use free association. They do not force the participants to use their imagination to develop ideas (although some may do so) but piggyback on the ideas of others thus participants tend to follow a more structured process.

Swann and Birke (2005) state that there are three critical aspects or characteristics of creativity: bisociation, autonomy and incubation. Bisociation is a combinatorial activity: it brings together different perspectives on the same issue. A distinction is made between the routine skills of thinking on a single plane, and the creative act which always operates on more than one plane. Bisociation is about perceiving an idea or situation, in two self-consistent but habitually incompatible frames of reference. Autonomy concerns the fact that the creative person needs to establish his own intellectual and creative independence. Incubation pertains to the fact that creativity usually does not take the form of sudden flashes of inspiration out of the blue. Rather, creativity is the culmination of long periods of sustained thought and effort - or incubation. This is not to deny that the final breakthrough may appear suddenly, but to emphasise that the breakthrough builds on long periods of painstaking thought.

\subsection{Creative Thinking Methods}

Brainstorming: McFadzean (1998b) notes that brainstorming relies on the absence of evaluation in the idea generation phase. Moreover, free-wheeling is encouraged so that an extensive list of ideas can be generated. The group members must be allowed to communicate an idea, however mundane, strange or wild, to the rest of the group. An idea that may seem impractical may contain a germ of a great solution. The facilitator meets the problem champion to develop a statement of the problem, to select the participants and to set up the meeting. The facilitator reiterates the problem statement to the group, sets out the ground rules, instructs the group on the purpose and process of brainstorming and conducts a warm-up exercise, if necessary. The facilitator asks the participants to generate possible solutions, without criticism, for about 30 to 45 minutes. The ideas are recorded on a flipchart by the facilitator, who must also encourage the group members to continue generating ideas. The facilitator leads the group back through the list of ideas encouraging them to combine statements and identify valuable ideas. The facilitator designates one person to receive any additional ideas that may occur to members after the meeting.

Brainwriting: McFadzean (1998b) argues that brainwriting not only maintains a form of anonymity thus reducing inhibitions, but it also allows parallel communication which negates domination by one or more individuals. Couger et al. (1993) note the distinction in brainwriting is the generation of ideas individually, and recording them on paper. The advantage over brainstorming is reduction of the effect of dominating individuals; brainwriting ensures that all participants have equal opportunity to share their ideas. The other advantage comes from the rotation of ideas among participants, with each person expanding and improving the idea. By the time the process is completed, everyone has ownership in the idea. It also tends to be a mature idea, ready to be implemented, compared to the results of brainstorming, where the ideas are mostly immature.

Synectics: Nolan (2003) notes that the synectics process views the initial approaches generated in a period of suspended judgement (similar to, but more sophisticated, than brainstorming) as starting points for further development, rather than as finished solutions to be accepted or rejected. They are called 'springboards': they are taking-off points, not (normally) end points. At the end of the explicitly divergent process to generate springboards, synectics moves on to the development process, which converts initially 
new, but not feasible approaches into new and feasible courses of action. It does so by using a developmental type of judgement, to be made by the individual who will be responsible for implementing the solution. A developmental judgement is a temporary, provisional judgement, which articulates what is, or might be, attractive about an idea and goes on to express its major shortcoming as a "need for improvement". There are two important elements to this apparently simple process: the first is the positive emphasis of finding, even searching for, all the value in the idea. This ensures that every idea that is evaluated is positively received, maintaining the safe climate created by the initial suspending judgement. There is no risk of rejection in expressing any idea. The second is the conversion of the one major negative into a "direction for improvement": "what I need now is a way to ...", inviting ideas directed at this new target. By continued iteration between the ideas and their constructive evaluation, the process moves (probably) to a course of action that is new and feasible and has the commitment of the individual who has been doing the directing.

Radial-diagram technique: Titus (2000) states that the radial-diagram technique is a simple, yet powerful, free-association technique, much like the traditional brainstorming approach. However, it proceeds by categorizing and graphically mapping out or linking related ideas and solutions together as they are generated. Thus, the radial-diagram technique operates very much like a structured version of the traditional brainstorming approach. The advantage of this approach is that the categorization process calls attention not so much to the specific solution, or idea generated, but rather to the category of solution generated. This ultimately helps students to identify the usefulness of even the most outlandish ideas (i.e., why the idea solves the problem), which, in turn, facilitates the production of more solutions that fall within that particular category. As with the case of traditional brainstorming, the radial diagram requires that one suspend judgement until all ideas have been generated.

Nominal group technique: Couger et al. (1993) note that the technique utilizes the positive features of both brainstorming and brainwriting. It uses silent generation of ideas in writing, round-robin recording of ideas, serial discussion for clarification, and subsequent rounds of writing. This approach reduces the inhibiting factors of both brainstorming and brainwriting, while retaining public sharing of ideas to stimulate new ideas.

Metaphors: McFadzean (1998b) states that metaphors can be used to create a fantasy situation so that a new perspective of the problem can be gained. There are a number of different types of metaphors that can be useful for problem solving and opportunity finding. These include metaphors of nature, vehicle metaphors, creational metaphors, the journey metaphor and so on. The group members are asked to write a brief statement of the problem. The facilitator asks the group to select a metaphor category or he can stipulate the category to the group. Each individual then needs to describe the situation using the metaphor category. The facilitator needs to stipulate whether the description should be of the present situation or the ideal situation. Using the descriptions developed by each team member, the participants can generate new ideas.

Object Stimulation: McFadzean (1998b) notes that object stimulation is an idea generation technique that can be used to explore the problem space as well as to enhance solution development. The technique encourages participants to view the situation from a different perspective by using unrelated stimuli. The group members are asked to develop a list of objects that are completely unrelated to the problem. Each individual then needs to select one object and describe it in detail. The group should use each description as a stimulus to generate new and novel ideas.

Manipulative verbs: Couger et al. (1993) argue that sometimes people need a kickstart to help them identify alternative approaches to solving a problem. The manipulative verb checklist provides words for kickstarting. The list also helps generate ideas by taking a verb from the list and checking the item against certain aspects of the problem, such as implementation. The comprehensive list of verbs helps reduce the possibility that a solution might be overlooked. Users of the technique sequentially move through a list of verbs to suggest possible solutions to the problem.

Heuristic ideation: McFadzean (1999) notes that, in the heuristic ideation technique, the problemsolving group develops a list of words that are completely unrelated to the problem. The group chooses two words and forces them together to stimulate ideas pertaining to the problem or situation. The purpose of such stimuli is to present a completely different problem perspective.

Suggested integration of problem elements (SIL): Couger et al. (1993) state that free association and forced relationships are combined to cause an integration of ideas until an acceptable solution is reached. Members of the group record their ideas in writing. Two people each read an idea. The remainder of the group tries to integrate the two ideas. Then another person reads his idea, and the group tries to integrate that idea with the first integrated result. The process continues, progressively adding an idea, until an acceptable solution is reached.

Morphological analysis: Couger et al. (1993) note that this is an electric, forced-relationship technique that consists of dividing a problem into its major parameters or dimensions and then subdividing these into 
different forms of the original dimensions. Various combinations of the subdivisions are then forced together and examined for possible solutions to the problem. Because of the systematic nature of the method, trial-anderror problem solving is avoided. The approach also causes ideas to be considered that might have been overlooked using a less systematic method.

Breakthrough Thinking: McFadzean (1998a) states that creativity can be enhanced by looking at the problem from a variety of perspectives and by breaking old mind patterns and forming new connections and perceptions. Breakthrough thinking also enable decision makers to view problems from a different perspective. It focuses on the future, that is the goals and purposes of the organisation or department, rather than what went wrong. This allows the decision-makers to view the problem from a different perspective and encourages the use of more creative problem solving techniques such as excursion. Here, participants look at colourful photographs or graphic words and try to link the images to the problem.

Guided Fantasy: McFadzean (1998a) states that, in guided fantasy, participants are asked to describe a scenario including their feelings, sounds, colours and smells. New ideas are developed from these scenarios and they are then linked back to the problem. Harkow (1996) argues that allowing students to visualize suggestions and providing them with guided fantasy as a prelude to creative writing experiences, can result in heightened creative output. As part of using guided fantasy to enhance creative writing in the classroom, after they had been presented with a fantasy prompt, students were allowed to visualize suggestions and then write their interpretations in the form of a story. When students were allowed to visualize, fantasize and even become the character in the story to be written they reacted in a more believable and creative way. Garfield et al. (2001) note that guided fantasy is an intuitive technique that instructs participants to use a fantasy world distant from the problem to help think of unusual ideas to solve the proposed problem. In their case each participant was given a description of a trip on a space shuttle as their fantasy world. This scenario provided an environment unrelated to the task as a stimulus for generating solutions to the proposed problem (campus parking space).

Wishful thinking: McFadzean (1998b) notes that wishful thinking as a creative problem solving technique forces participants to look at a "perfect future". By using this method it allows group members to develop a goal that can be attained. The group members are asked to write a brief statement of the problem. The facilitator tells the group to assume that everything is possible. Each individual then needs to develop some fantasy statements about the future using terms such as: In the future, it would be nice if the organisation did... What really needs to happen to be a great company is... If I were in charge of this situation I would do... The group members must then examine each fantasy statement and develop ideas on how these can be achieved.

Rich pictures: McFadzean (1998b) states that, with rich pictures, group members are asked to write a brief statement of the problem. The facilitator then asks each individual to draw two pictures. The pictures may be a metaphor of the situation, for example a vehicle or an animal. The first drawing would be a picture of how each participant would like to see the situation in the future. The second picture would be a drawing of how the participants see the present situation. Each participant is asked to describe the picture of the present first. Not only should he describe the picture but a description should also be given of the properties of the objects drawn and why they have been drawn that way. Next, a description of the picture of the future should be given. Again, the properties and the relationships of the objects should be described. From the descriptions given by the participants new ideas can then be generated.

Force field analysis: McFadzean (1999) states that force field analysis was designed to help users to identify factors that contribute to the problem or impede the implementation of its solution. A problem statement is developed and written on a flip chart. Each individual is asked to write two scenarios: a description of what the situation would be like if a complete catastrophe were to occur; and a description of the ideal situation. All the catastrophic and ideal scenarios are arranged on a continuum with a center line drawn between them. The group members are asked to list the forces that could contribute to making the situation catastrophic and those that will make the situation ideal. Force field analysis can stimulate creativity by helping users to define a vision and identify strengths that need to be enhanced and weaknesses that need to be reduced.

Associations/images: Couger et al. (1993) note that the technique builds on the natural inclination of humans to associate. The linking or combining process is another way of expanding the solution space. The leader assists the group in identifying the problem or opportunity to be examined. The leader asks the participants to select a solution to the problem phrased in the form of a goal or wish. The leader picks a key concept phrased in the form of a goal or wish. The leader picks a key concept that is central to the goal/ wish statements. The leader asks the group to think of a world that is remote from the world of the problem. The leader requests the group to lay aside both the problem and the goal/ wish developed previously, and to list associations and images that characterize the remote world. The leader directs the group to relate the list of associations and images of the remote world to the world of the problem. The leader directs the group to 
develop second generation associations and images from any one of those listed before, extracting key principles and applying them in a more realistic way without diluting the innovation. The group selects and implements appealing ideas developed.

Wildest idea: Couger et al. (1993) state that the technique asks people to try to come up with a wild idea. A wild idea is selected. With this as starting point, the group continues to generate ideas. If no practical ideas emerge, another wild idea is used, and the process continues until an acceptable idea is found.

Peaceful setting: Couger et al. (1993) note that the objective is to enable people to mentally remove themselves from present surroundings so they can have access to a less cluttered, more open mental process. The goal is to try to eliminate the constraints of the normal work environment that impede full use of their native creative ability. By trying to use all five senses in this setting, they can more easily call in their sixth sense, intuition.

Progressive abstraction: Titus (2000) states that the progressive abstraction technique is designed to expand the scope of the problem by redefining it at progressively higher levels of abstraction. The process of moving to higher levels of abstraction helps to reveal the multi-dimensional nature of the problem and ultimately expands the solution set. The procedure begins with an initial problem and progresses by exploring the consequences of not resolving the problem. In essence, what other problems will surface if one is unable to find an acceptable solution to the initial problem. The procedure is then applied to the new resulting problem(s) and continues until no new problems surface.

Lotus Blossom technique: Titus (2000) notes that the Lotus blossom technique form part of a class of techniques designed to systematically uncover and question the basic assumptions and constraints surrounding the problem. It is designed to systematically identify and list all of the assumptions held about the problem. Employing this technique provides the basis for knowing which assumptions to question or verify, thereby directing what information needs to be collected. It proceeds by first listing the problem statement and then identifying and grouping assumptions about the problem into related categorizes.

Bug list technique: Titus (2000) argues that the bug list can best be described as a fault-finding approach to identifying new problems and opportunities. It enhances problem sensitivity by heightening individuals' awareness of their dissatisfaction with existing things. The bug list procedure requires individuals to identify and list those things that irritate or bug them about some object. When used with a group, a consolidated list of the common group bugs is compiled and voted upon to identify the things that most irritate the group. The bug list technique is effective because, every dissatisfaction is a signal that some need is not being met.

Interrogatories - 5Ws and H technique: Couger et al. (1993) argue that the who-what-where-whenwhy and how questions aid in expanding an individual's or group's perspective of a problem or opportunity. The technique provides a simple, easy-to-remember framework for systematically gathering data that are relevant to identifying and solving the problem. By going through several cycles of the $5 \mathrm{Ws} / \mathrm{H}$, alternatives related to the problem/ opportunity can be explored exhaustively.

Problem reversal: Couger et al. (1993) note that the technique is valuable for defining a problem in more solvable terms and for finding solutions to everyday problems. The problem statement is written down in question form. The verb or action content of the statement is identified. The meaning of the verb or action content is reversed and the problem is restated in question form. Answers to the reversed problem statement is listed, and again reversed.

Assumption Reversal: Hender et al. (2001) note the phases of the assumption reversal idea generation technique are: 1) list all the major assumptions about the problem; 2) reverse each assumption in any way possible; 3) using the reversals as stimuli, generate ideas. The procedure of listing assumptions and then reversing them is the external stimulus, which is the means of changing perspective. This invokes a moderate perspective change. These reversed assumptions, however, are still related to the problem. The link back to the problem and subsequent idea generation is by free association.

Attribute association: Couger et al. (1993) state that the attribute association technique works on the premise that all ideas originate from previous ideas that have been modified in some way. The socket wrench, for example, is an improvement on the wrench. The ratchet screwdriver evolved by changing one of the attributes of the traditional screwdriver to one of pushing instead of turning the handle. The problem and its objectives are stated. All the characteristics of a product, object, or idea related to the problem are listed. The characteristics or attributes are systematically modified to meet the objectives.

Dimensional analysis: Couger et al. (1993) note that the technique is designed to clarify and explore the dimensions and limits of a problem. It examines five elements of a problem: substantive dimension, spatial dimension, temporal dimension, qualitative dimension, and quantitative dimension. It is useful in forcing a problem solver to consider the many implications associated with the various problem dimensions. The best 
use of the technique is as a checklist for pre-problem solving, that is, a general guideline preceding the use of some other analytical method.

Boundary examinations: Couger et al. (1993) argue that the act of defining a problem involves certain assumptions about problem boundaries. Boundaries determine how information is gathered, organized, and processed. Boundary rigidity will affect problem-solving success. Boundary examinations are based upon the assumption that a problem's boundaries are neither correct nor incorrect. The objective is to restructure the assumptions of a problem to provide a new way of looking at at. The major strengths of the technique are its potential for 1) producing more provocative problem definitions, 2) clarifying often indistinguishable problem boundaries, 3) demonstrating the importance of formulating flexible problem definitions, and 4) coping with management teams that are overly precise in their problem definitions.

Disjointed incrementalism: Couger et al. (1993) state that the technique is useful for situations involving complex decisions and vaguely defined, changing objectives. The disjointed aspect of the technique refers to the way in which many policy problems are analyzed at different times in different locations without any apparent coordinating efforts and without the benefit of relevant past experience. Incrementalism refers to the prescriptions used to compare differences or increments in the consequences of various alternatives with one another and with a present (state) situation. Thus, decisions are made by evaluating the trade-offs possible between an increment of one value and an increment of another value. The technique has the ability to reduce information processing demands made upon a problem solver. It allows a systematic way to restrict the number of alternatives and consequences requiring consideration. The technique can decrease the complexity and uncertainty of a decision environment.

Decomposable matrices: Couger et al. (1993) note that the technique builds on a view that complexity in the world has evolved from simple structures into progressively formal hierarchic systems. To understand complexity, complex hierarchic systems can be analyzed using a basic property of their structure: near decomposability. The concept of near decomposability refers to the fact that the subsystems of some hierarchic systems maintain some, although not total, interdependence from other subsystems. Decomposable matrices are especially useful for highly system-based problems. The components of each subsystem are listed and arranged within a matrix. Weights are assigned to each of the interactions. Relationships between components can then be selected as the focus for generation of problem solutions. Because this technique forces identification of problem subsystems, their components, and how they interact, it can cause the emergence of a clearer picture of important problem elements.

\subsection{Analytical Thinking}

Henrich (2014) argues that, in the context of national culture, various lines of evidence suggest that greater individualism and more analytical thinking are linked to innovation, novelty, and creativity. Thinking analytically means breaking things down into their constituent parts and assigning properties to those parts. Similarities are judged according to rule-based categories, and current trends are expected to continue. Holistic thinking, by contrast, focuses on relationships between objects or people anchored in their concrete contexts. Similarity is judged overall, not on the basis of logical rules. Trends are expected to be cyclical.

Ward (2004) notes that some creativity models include steps such as problem construction, problem definition, and problem discovery. Implicit or explicit in these models is the belief that the way in which people conceptualize a problem strongly influences their likelihood of achieving an original or creative solution. Hender et al. (2001) note that experiments have found that decomposing the problem into separate sub problems, each having its own named dialog, results in the generation of ideas of a higher quantity and quality than those generated when the problem is left intact. This is because problem decomposition expands the problem space by focusing attention over the entire problem. In a similar way, thinking of possible causes of the problem before brainstorming expands the problem space and leads to the generation of more ideas. Baron (2007) argues that pattern recognition appears to be closely related to opportunity recognition in the domain of entrepreneurship. This process involves noticing meaningful patterns in complex events, trends, or changes. In essence, it includes 1) recognizing links between trends, changes, and events that appear, at first glance, to be unconnected, and 2) noticing that these connections form an identifiable pattern.

Sternberg (2006) argues that creativity and simply thinking in novel ways are facilitated when people are willing to put in up-front time to think in new ways. It is found that better thinkers tend to spend relatively more time than do poorer reasoners in global, up-front meta-componential planning when they solve difficult, novel-reasoning problems. Poorer reasoners, conversely, tend to spend relatively more time in local planning. Presumably, the better thinkers recognize that it is better to invest more time up front so as to be able to process a problem more efficiently later on.

Leron and Hazzan (2009) note that, according to dual-process theory, human cognition and behaviour 
operate in parallel in two quite different modes, called System 1 (S1) and System 2 (S2), roughly corresponding to common sense notions of intuitive and analytical modes of thinking. These modes operate in different ways, are activated by different parts of the brain, and have different evolutionary history (S2 being evolutionarily more recent and, in fact, largely reflecting cultural evolution). The distinction between perception and cognition is ancient and well known, but the introduction of S1, which sits midway between perception and (analytical) cognition, is relatively new and has important consequences for how empirical findings in cognitive psychology are interpreted, including the wide-ranging rationality debate. Like perception, S1 processes are characterized as being fast, automatic, effortless, unconscious and inflexible (hard to change or overcome); unlike perceptions, S1 processes can be language-mediated and relate to events not in the hereand-now. S2 processes are slow, conscious, effortful, computationally expensive (drawing heavily on working memory resources), and relatively flexible. The two systems differ mainly on the dimension of accessibility: how fast and how easily things come to mind. In most situations, S1 and S2 work in concert to produce adaptive responses, but in some cases (such as the ones concocted in the Heuristics-and-biases and in the reasoning research), S1 generates quick automatic non-normative responses, while S2 may or may not intervene in its role as monitor and critic to correct or override S1's response. Many of the non-normative answers people give in psychological experiments can be explained by the quick and automatic responses of S1, and the frequent failure of S2 to intervene in its role as critic of S1.

Leron and Hazzan (2009) continue that what is part of human nature need not be innate: humans are not born walking or talking. What seem to be innate are the motivation and the ability to engage the speciestypical physical and social environment in such a way that the required skill will develop. Humans do manage to learn many modern skills (such as writing or driving, or some mathematics), because of the mind's plasticity and its ability to "co-opt" ancient cognitive mechanisms for new purposes. Human nature consists, by definition, of a more-or-less fixed collection of traits and behaviours that all human beings in all cultures acquire spontaneously and automatically under normal developmental conditions. In their view, System 1 contains all the traits and behaviours that comprise human nature but, on top of that, also all the traits and behaviours that have become S1 for a particular culture or a particular person because of specific (nonuniversal) developmental conditions. For example, learning language is part of human nature and, thus, part of S1 for all human beings under normal developmental conditions; in contrast, speaking English is not part of human nature but is an S1 skill for people whose mother tongue is English. Similarly, driving a car is not part of human nature but has become an S1 skill for experienced drivers, as evidenced by their ability to hold an intellectual conversation (an S2 task, fully engaging the working memory resources) while driving.

In examining mathematics education theory and practice, and mathematical errors, Leron and Hazzan (2009) conclude that mathematical errors arise, because people do not look deeply into the problem but, instead, do some routine calculations based on verbal cues. Also, people tend to personalize the problem ("if I tested positive..."), bringing in a whole baggage of realistic conditions that are abstracted away in the original formulation. Furthermore, in some cases, "the dormant S2" failed to catch the error in its role as critic and monitor of S1's output. The issue lies in the complexity of what S2 is required to notice and correct - the effort and skill required. It appears that heuristic processes cannot lead to correct integration of diagnostic and base rate information, and so certain (Bayesian) problems can only be solved analytically.

Epstein et al. (1996) note that psychologists from various persuasions have proposed two fundamentally different modes of processing information: one that has been variously referred to as intuitive, natural, automatic, heuristic, schematic, prototypical, narrative, implicit, imagistic-nonverbal, experiential, mythos, and first-signal system; and the other as thinking-conceptual-logical, analytical-rational, deliberativeeffortful-intentional-systematic, explicit, extensional, verbal, logos, and second-signal system. Appeals to emotions, personal experience, and the use of concrete examples could be more effective for individuals who process information primarily in an intuitive mode, whereas presenting facts and logical arguments could be more effective for individuals who process information primarily in an analytical mode. The global theory of personality referred to as cognitive-experiential self-theory (CEST), proposes that people process information by two parallel, interactive systems: a rational system and an experiential system. According to CEST, the rational system operates primarily at the conscious level and is intentional, analytic, primarily verbal, and relatively affect free. The experiential system is assumed to be automatic, preconscious, holistic, associationistic, primarily nonverbal, and intimately associated with affect. Heuristic processing represents the natural mode of the experiential system. Behaviour and conscious thought are a joint function of the two systems. The systems normally engage in seamless, integrated interaction, but they sometimes conflict, experienced as a struggle between feelings and thoughts.

Rusou et al. (2013) note that there is considerable agreement among researchers that information in decision making involves two qualitatively different thinking modes: 1) an intuitive mode characterized by 
fast and parallel processes that are affective, holistic, and associative in nature, and 2) a deliberative/ analytical mode characterized by slower processes that are rule based in nature. Despite the above-mentioned agreement, there are different viewpoints regarding the ways in which these two thinking modes interact. Identifying the circumstances under which each thinking mode is preferable might help in understanding the advantages of each mode. In this respect, several researchers have highlighted the importance of one moderating factor, which is the compatibility between thinking mode and task characteristics. The nature of the task could be more intuitive or more analytical, depending on task characteristics. The characteristics of intuitive tasks include high familiarity, pictorial presentation, subjective measure, and unavailability of an organizing principle or algorithm to integrate cues. Analytical tasks are characterized by quantitative presentation, objective measures, and an organizing principle readily available. Analytical deliberation is adequate for tasks that involve objective, easily verbalized attributes but is less adequate for tasks that involve a large affective component or in which the dimensions of the stimuli are ill-defined. The intuitive mode is more adequate when nonverbal information and/ or concrete stimuli are involved, whereas the analytical mode is more adequate when abstract problems are solved through symbols (numbers and words) and logical inference. The intuitive mode is used when processing emotion-arousing stimuli that represent events in a manner similar to how they are experienced in real life. Tasks are more likely to be processed through the intuitive mode when their context and form promote visual reasoning. Tasks in which participants must follow strict rules are performed best through analytical deliberation.

Mihov et al. (2010) state that the brain's right hemisphere is better at exploring for new possibilities while the left hemisphere is more likely to result in negative or positive transfer (the application of a previously learnt concept or pattern to a new problem). A further conclusion is that the right hemisphere is better at semantically connecting verbal phrases rather than exploring their direct meaning. A review of the literature also points out several findings that suggest the superiority of the left hemisphere. One moderator existing in the literature that examines the relative dominance of the right hemisphere in the processes associated with creativity, is the modality of the task (lexical or figural). In these studies, a large number of the creativity tasks are in fact lexical in nature. It is, thus, very likely that: 1) if there were bilateral activation or no relative hemispheric dominance, it could be due to the left hemisphere being activated to process the information (and not necessarily to contribute creatively to the task solution); and 2) if there were relative left hemispheric dominance, it could be that the task requires so much verbal processing that the right-hemisphere activation is overshadowed by the lexical complexity. Thus, they hypothesize that studies that show relative left hemispheric activation, verbal tasks will be a significant predictor.

In reflecting on teaching writing, Sams (2003) note the process she has shared with her students since that first year of teaching, consists of analyzing sentences through a process of questioning that reveals the precise relationship of every part to the whole. By the time students have worked through the sequence of sentence types, they have not only developed a deep understanding and conscious awareness of sentence patterns, but they are able to "see" groups of words as moveable and replaceable parts, and they understand the changes in meaning and emphasis that occur by adding, deleting, rearranging, and reforming the parts. Thorough analysis accomplishes in depth what sentence combining only touches upon, for sentence combining succeeds in drawing attention to some structures that can be used for expanding a sentence, but it provides neither method nor rationale for choosing one structure over others. Nor does it instil an understanding of the language system as a whole that gives students the control over structures they need. The thought process is not a simple matter of word choice, of finding the precise word. The writer must be able to see the units that are being joined, identify how they are joined, identify the precise relationship between them, be aware of the variety of options that exist for expressing that relationship, and choose the one that best fit his meaning. Students who are taught that words, phrases, and sentences bear specific relationships to one another and who are taught to carefully analyze these relationships develop an enhanced ability to recognize weak links in their own and others' writings. A language unit - a word, phrase, clause, sentence, or paragraph - can be examined in one of three ways: 1) in isolation from other units, as an entity in and of itself (a particle); 2) in terms of its relationship to a unit with which it blends (a wave); 3 ) in terms of its relationship to other units of the larger ordered system of which it is a part (the sea, or the linguistic field).

Robbins (2011) notes that, in the solution of a difficult problem, mathematical or otherwise, people resort to overt responses, vocal or written. In the context of talk aloud problem solving, students eventually acquire both repertoires and learn not only to speak, but to listen to themselves and adjust their performance based upon what they hear. The Problem Solver produces a goal directed dialogue, while the Active Listener evaluates the dialogue for its progress in meeting the problem solving goal. The students have learned to listen to themselves, evaluate their own effort, and provide verbal feedback that prompts further action and eliminates alternatives. Sometimes the speaker or Problem Solver, is unsure or appears to lack confidence; that is, the 
learner may emit a weak, hesitant, or incomplete response. The Active Listener, who is the same person as the Problem Solver, may hear the response and add further dialogue, that prompts yet other responses. It is at those moments when the Problem Solver's response is weak, perhaps having forgotten a detail, that the same person as listener will be able to recognize the correct response even though there was a hesitation in its production. The reasoning process may also make use of a series of formal or thematic prompts; reading the text of the problem aloud again may provide an opportunity to inspect the text for the required information. Students are taught to change the emphasis, to stop at particular words that are key to the meaning.

Robbins (2011) continues that analytical thinking is necessary when an ambiguous situation requires the learner to identify or create a problem to solve. It involves the reasoning process described before, but involves a further element of inquiry, often in situations with less well-defined parameters and outcomes. This skill is required when a learner faces an often ill-defined, more global problem. Where the problem is not clear, the strategy required is one of inquiry, and to inquire is to question. Questioning combined with reasoning, thus, is the key to analytical thinking. In John Dewey's words: "Thinking is inquiry, investigation, turning over, probing or delving into, so as to find something new or to see what is already known in a different light. In short, it is questioning." Questioning must be taught separately and added to the learner's repertoire to produce a true analytical thinker.

Freshwater and Avis (2004) argue that the creative and inventive aspects of analysis and interpretation should be acknowledged. They propose that the logic of hypothetical reasoning allows just such scope for innovation. If the nature of analysis is examined more closely, it can be said that it is a process of breaking down - it is a reductive process. They further propose that the logical structure of critical reflection upon evidence is based on hypothetical reasoning. Hypothetical reasoning involves building hypotheses as plausible ways of organizing evidence from which testable consequences can be deduced. Simply stated, hypothetical reasoning starts from the question: if this hypothesis could be justified then what consequences might be expected for the observations and theories? The outcome of considering this question should be regarded as a set of evidence-based hypotheses that are proposed rather than asserted; whether they contribute to knowledge can be evaluated in terms of pragmatic criteria, such as convenience and simplicity, when making sense of experience. Analysis uses supposition to produce a selective reading, a synopsis, one that preserves the basic structures whilst making new possibilities apparent. The reflective processes of refining questions, selecting events and observations for inclusion, coding and identifying themes are all integral aspects of analysis, but these can only be achieved through the application of a working hypothesis.

Freshwater and Avis (2004) further state that, as empirical evidence is subjected to critical reflection, the analyst develops hypotheses in order to produce patterns and commonalities through a process of reduction. The meaning of these themes and commonalities is explored by attempting to weave them into existing theory or by building new hypotheses that allow them to fit with existing theory. However, the hypotheses that permit the reduction and interpretation are conditional and are held, at first, without conviction. They are used as convenient explanatory beliefs that may be inchoate and vague, but as the process of analysis and interpretation proceeds, the analyst attempts to give these beliefs more determinate content and specific connections with surrounding theory. An integral aspect of hypothesis testing includes prediction. The analyst working with an explanatory hypothesis will attempt to predict themes and commonalities to be observed within the evidence, and in future experience. Critical reflection on evidence has a common underlying logic based on hypothetical reasoning.

\subsection{Analogical Thinking}

Hargadon and Bechky (2006) argue that individuals facing problematic situations find solutions through a process of analogical reasoning, of trying to make sense of a new situation (the target analog) by relating it to a more familiar one (the source analog). Analogical problem solving occurs when an individual recognizes similarities in the new situation to old problems (and their solutions) that he has learned in the past. Transferring these existing solutions from old problems provides a solution to the new problem. Ward (2004) argues that analogy, or the mapping of knowledge from a familiar domain to a less familiar one, is central to creative developments in science, art, music, and literature and may also have applicability to entrepreneurship, as when a new successful venture is based on the principles that operate in other current successful ventures. Holyoak and Thagard (1997) describe thinking by analogy: trying to reason and learn about a new situation (the target analog) by relating it to a more familiar situation (the source analog) that can be viewed as structurally parallel. It is also shown how analogical reasoning can be used to predict or describe possible outcomes, scenarios, or characteristics.

Jee et al. (2010) note that, in the geosciences, analogies are extremely common, perhaps because so much of the field deals with processes and forces that cannot be directly perceived and thus can often be known 
only through comparison to something else. Such analogies often serve as frameworks for knowledge. There are three main steps of the processes of analogical comparison: 1) retrieving knowledge about the examples in the analogy, 2) comparing the two examples on the basis of the relationships that they have in common, and 3) making inferences about the examples based on their common relations. Learning from a physical model also involves projective analogy - in this case projecting observations and facts about an available source model to an unfamiliar target object or system. Another important kind of analogy, referred to as mutual alignment analogies, is when the source and the target are both only partially understood. In these analogies, the two analogs are typically both from the same domain or topic and are similar enough to be easily alignable. Mutual alignment analogies can be quite powerful, because they can help students understand both examples better. In projective analogy, the goal is not only to notice common structure, but to import further structure from the familiar to the new topic. In contrast, in mutual alignment analogies, the chief goal is precisely for the student to notice and abstract the common system. Another use of mutual alignment is for contrast. Once the two examples are aligned, differences connected to the common system stand out.

Richert et al. (2005) note that psychological research on analogical thinking traditionally draws a distinction between source and target analogs. Analogies postulate links between a particular object, event, or state of affairs (the "target" analog) and some previously independent object, event, or state of affairs (the "source" analog). Analogical reasoning has been defined as "the process of understanding a novel situation in terms of one that is already familiar", suggesting that source analogs consist of familiar situations and target analogs consist of novel ones. This definition has serious drawbacks, however. Some instances of analogical thinking are based on the recognition of connections between two situations that are both novel or that are both familiar. They maintain that what is characteristic of spontaneous analogical thinking is that the connections it proposes are novel, insofar as the person is drawing parallels that he has not recognized before (even if others have already done so at least partly by independent means). There are three general constraints that guide the selection of analogical comparisons. These constraints are similarity, structure, and purpose.

Bonnardel and Marmèche (2004) note that analogy-making is usually described as allowing two kinds of analogies: 1) intradomain analogies, when the target (e.g. the situation or problem at hand) and the source (a previous similar situation) belong to the same conceptual domain; and 2) interdomain analogies, when the target and the source belong to different conceptual domains. In addition, relationships are established between the target and the source. Intradomain analogies would be based on both surface similarities and structural similarities between the target and the source, whereas interdomain analogies would be based only on structural similarities (or underlying principles) between the target and the sources.

Gassmann and Zeschky (2008) notes that analogical thinking happens if a familiar problem is used to solve a novel problem of the same type. Non-obvious analogies may entail highly novel solutions because the combination of more distant pieces of knowledge is associated with higher innovative potential. From cognitive psychology perspective, analogical thinking entails the transfer of knowledge from one domain that usually already exists in memory to the domain to be explained. Management scholars have argued that the use of analogies typically includes the transfer of knowledge, where knowledge acquired in one situation is applied to another. Similarity of some basic elements between the source where the problem origins (i.e. the problem source) and the source where the analogy is found (i.e. the solution source) is a vital precondition for analogies to be identified. Similarity has also been described in a continuum from "near" or "surface" analogies to "far" or "structural" analogies. Near analogies are much easier identified than far analogies, as near analogies often entail obvious surface similarities such as similar design, while far analogies typically entail similarities in the structural relationships between source and target attributes. Near and far analogies require different types of information to be mapped and transferred. With near analogies both surface-level attributes and relations between the attributes are mapped and transferred, while the lack of surface-level attributes of far analogies leaves the mapping to occur between common relations. The identification of far analogies requires the identification of similarities in the relational (vs. surface) structure between the problem and the solution source, which is often difficult when surface similarities are completely absent. However, if successfully implemented, far or structural analogies serve as the base for "mental leaps" and can lead to radical innovation. abstracting the problem by in-depth technical and contextual analysis is pivotal when searching for analogical solutions.

Gentner (2010) states that, according to structure-mapping theory, human comparison involves a process of establishing a structural alignment between two represented situations and then projecting inferences. The commonalities and differences between two situations are found by determining the maximal structurally consistent alignment between the representations of the two situations. As a natural outcome of the alignment process, candidate inferences are projected from the base to the target. These inferences are propositions connected to the common system in one analog, but not yet present in the other. Achieving a 
structural alignment sets the stage for four kinds of learning: abstraction, contrast, inference-projection, and re-representation. In abstraction, the common system resulting from the alignment becomes more salient and more available for future use. In contrast, alignable differences - differences that occupy the same role in the two systems - are highlighted. Inference-projection occurs when one member of the pair is more complete in its structure than the other; in this case, spontaneous candidate inferences will be made that enrich the lesscomplete item. A further way that learning can occur is re-representation: If there is good reason to believe two (non-identical) relations should match (e.g., a very good overall structural match), then one or both of the nonmatching predicates may be re-represented to permit the overall match.

\subsection{Imaginary Thinking}

Zeki (2001) states that how the brain forms abstractions is a central problem in cognitive neurobiology. Through a process that is only now beginning to be physiologically charted, cells in the brain seem to be able to recognize objects in a view-invariant manner after brief exposure to several distinct views, which they obviously synthesize.

Pelaprat and Cole (2011) propose a theory of imagination as a biologically required, culturallymediated, temporally-variable process - and not as a frozen function. They characterize imagination as a "gapfilling" process. While "images" are the catch-all term for the products of imagination, the image is itself heterogeneous and heterochronous, since it depends on the conditions and constraints in which the process of gap-filling unfolds; that is, on the conditions on which a stable image can be produced by an individual who begins to sense their self as a self in that world. It is widely assumed that imagination comes into play only when an object is absent from the senses. This starting point also unwittingly assumes that "presence to the senses" is a condition of perception. Existing evidence indicates that both assumptions are untenable and that the reduction of imagination to perception or fantasy is mistaken. Imagination is present in a primitive, yet clear form, even when an object is present to the senses (the argument here is focused on vision, but the point is believed to be quite general). This alternative conception compels an understanding of imagination as a basic, pervasive, and distributed faculty of materially embodied thought and action, as opposed to a marginal, specialized activity of the mind. In their view, the physiology of perception warrants the conclusion that it is incorrect to think of imagination as a process occurring only in the absence of the imagined object. Instead, it seems necessary for human cognition that individuals constantly engage in a process of image formation. Image formation is the "connecting bridge" between two states of experience: one conditioned by the phylogenetic history of the human species, the other by the cultural-historical environment and history of experience. What this bridging process entails - and what the results of the fixed image experiment indicate is that human beings are by nature always engaged in a process of image formation - of imagination. They are constantly engaged in this image formation process because, in fact, they are situated between nature and culture; both act as constraints, as principles that make thought possible, on the condition that a third term the individual's "resolving activity," or "gap-filling" - is involved.

Vygotsky (1991) describes pathological cases and notes that what is seen in these cases, in a clear, sharp and extremely graphic form, may be regarded as representing a complete anti-thesis to fantasy and creativity. There exists examples of a form of behaviour in which there is a complete absence of any elements of imagination and creativity. A person who is capable of pouring himself a glass of water from a carafe when thirst motivates him to do so, but who is incapable of performing the same action at another time, or a person who cannot repeat a sentence which says that the weather is bad when the weather is good: All this tells a lot which is significant and fundamental for the understanding of what underlies fantasy and creativity, and what links them to those higher intellectual functions which have become disturbed and disrupted in such cases. It can even be said that the behaviour observed in these patients is striking, above all, by the fact that it is not free. The person cannot do something which is not directly motivated by an actual situation. What appears to be beyond his ability is to create a situation or to modify it in such a way as to become free of the immediate influence of external and internal stimuli. Herder's thesis states that the language of thinking is the language of freedom (Vygotsky, 1991:269). Gelb develops this idea further when he says: "It is only man who is capable of doing something which is senseless" (Vygotsky, 1991:269). This illustrate the straight-forward idea that imagination and creativity are linked to a free reworking of various elements of experience, freely combined, and which, as a precondition, without fail, require the level of inner freedom of thought, action and cognizing which only he who has mastered thinking in concepts can achieve.

Vygotsky (1991) continues that it is not just that the adolescent experiences a severe distortion of the relative proportion of things, and that the structures of the intellectual functions are transmitted in an inaccurate form, but that the very process of imagination and creativity acquires a wrong interpretation. This false interpretation of fantasy is due to it being viewed one-sidedly, as a function which is linked to emotional life, 
the life of inclinations and sentiments; but its other side, which is linked to intellectual life, remains obscure. But, as Pushkin has aptly remarked, "imagination is as necessary in geometry as it is in poetry" (Vygotsky, 1991:270). Everything that necessitates artistic transformation of reality, everything that leads to inventiveness and the creation of anything new, requires the indispensable participation of fantasy. In this sense some writers quite rightly contrapose fantasy as creative imagination to memory as reproductive imagery. Whilst thinking in concepts is characterized by its existence in the realm of the abstract and general, imagination exists in the concrete sphere.

Vygotsky (1991) further states that it would not be quite correct to regard the function of fantasy as an exclusively visual, imagistic and concrete activity. Quite rightly, it has been pointed out that the same sort of visual quality is also characteristic of the imagery of memory. On the other hand, activity of a schematic or barely visual type is also present in fantasy. According to Lindworsky (Vygotsky, 1991:274), if we limit fantasy exclusively to the realm of visual conceptions, and remove any aspects of thinking from it, then it would not be possible to describe a poetic creation as a product of the activity of fantasy! In exactly the same way, Meumann disagrees with Lau's point of view, who saw the difference between fantasy and thinking in the fact that the former operates in visual images and does not contain any elements of abstract thinking (Vygotsky, 1991:274). Meumann states that elements of abstract thinking are never absent from our images and perceptions. And there is no way in which they could not be there, because in an adult the entire conceptual material exists in a form which has been reworked by abstract thinking. Wundt has also expressed the same idea when he objected to fantasy being regarded as simply the work of visual conceptions (Vygotsky, 1991:274).

LeBoutillier and Marks (2003) state that, in the last three decades, there has been a steady flow of empirical papers investigating the role of mental imagery in the creative process. Although a diverse range of inventive protocols has been developed, two standard approaches dominate the research literature. The first of these, the individual differences approach, focuses upon a hypothesized association between self-reported mental imagery and divergent thinking measures. The second, the image generation approach, employs protocols that investigate the emergence of creativity through the visualization of specific forms. A review of the individual differences approach to mental imagery and the creative process suggests that the main stimulus for empirical research of this kind is the anecdotal evidence provided by historically creative individuals. Reports of the association between mental imagery and creativity in the development of history-making ideas abound. Shepard (1978) emphasizes the importance of the voluntary manipulation of mental images (e.g. the mental rotation of stimuli). He provides non-empirical support through reports of the use of mental transformations by creative people. Suler (1980) believes that creativity follows from the emergence of an unconscious flow of imaginings (primary process thinking).

LeBoutillier and Marks (2003) continue that, even though reports of the association of mental imagery with creativity vary in style, form, content, and context, the empirical research has adopted two fairly stable protocols. The most frequently employed of these, the individual difference approach, pays scant attention to the explanatory accounts of the use of mental imagery in creativity. Rather, it seems to be grounded in the observations of creative individuals and simple threshold notions. Based on the assumption that mental imagery and creativity are continuous variables, the basic tenet is that differing degrees of imagery ability (and possibly additional prerequisite attributes or contexts) reflect differing creativity abilities. A review of papers employing the individual differences approach reveals an association principle. That is, the protocol searches for degrees of association between mental imagery and creativity variables. The measures used in the approach are roughly summarized as: 1) a self-report measure(s) of an aspect of mental imagery (normally control or vividness); 2) a test(s) of divergent thinking which provides individual measures of fluency, flexibility, and originality; 3 ) an intervening variable that is hypothesized to clarify the association between measures 1 and 2 (e.g. intelligence, verbal ability, cognitive style, instruction to image).

LeBoutillier and Marks (2003) note that the reports of Faraday and Einstein lay claim to an atypical non-verbal thinking. Also, a large proportion of historically creative individuals report having synaesthesia, a form of mental imagery reported in only $20 \%$ of the population. It is also noted that, even though extraordinary individuals all used their mental images in extraordinarily creative ways, the actual images themselves did not differ in extraordinary ways. They note that mental images, and their reported associations to creativity, are found in such a variety of forms that it would not be reasonable to assume a defining feature of mental images in creativity.

Folkmann (2010) sees imagination as the capacity or the faculty of consciousness to envision things that are not present in the physical world that surround us. "Imaging" and "imagery" is not something intangible which takes place in a mysterious "medium", i.e. the mind; instead "imaging is a doing" that alludes to the thinkable, and this means: to the do-able. 
Antonietti and Colombo (2011) state that mental images - a kind of representation people often employ in everyday life - play a facilitating role in thought processes as a means of simulation and as a means of symbolization. With simulation, the images allow a person to anticipate mentally the actual operations and the physical changes and provide an internal representation that keeps analogical correspondence with the outside world. With symbolization, mental images stand for objects or concrete events, which are replaced by conventional signs. In the first case, images are useful because they offer the opportunity to view the mental consequences of the situation that the representation in verbal or abstract terms does not make immediately obvious. In the second case, the images help one to mentally manipulate the elements of a situation, because mental images require less memory load than other representations, thus allowing smooth and rapid transformations of elements.

Antonietti and Colombo (2011) note that, with more specific reference to creativity, the search for similarities and differences and the identification of links between distant realities are facilitated by mental images that are sensitive to structural symmetries and organizations. These mental images allow one to modify data so that the changes which are to be produced in reality may be more flexibly simulated in the mind. Furthermore, mental images allow one to reorganize the way in which one represents a situation so that it can be reconsidered in a more productive manner. Finally, the mental representation of information in visual form can help one by providing a pictorial form for abstract concepts, thus allowing simultaneous representation of various elements of a situation which thereby facilitates the identification of relationships between the various elements. Mental images can therefore help the creative process because the representation is particularly flexible, easily convertible, and useful to combine multiple elements into a new concept.

Couger et al. (1993) note that Albert Einstein used what he called mental experiments to stimulate new perspectives and ideas. He once imagined himself as a tiny being riding through space on a ray of light, which helped him develop his general theory of relativity. Heppner et al. (1994) argue that guided imagery in career counselling is a structured activity in which the counsellor provides guidelines to spur the imaginations of their clients. Internal images can be a motivational and inspiring way to capture occupational daydreams that clients may frequently experience. Daydreams concerning one's career are often predictive of future vocational choice. Guided imagery is divided into the following three distinct parts: 1) inducing a relaxed state, 2) describing the fantasy itself, and 3) processing the fantasy. The actual image developed for the career client can take any form. For example, the counsellor may assist the client in imagining a day at work that takes place 10 years in the future. The counsellor could provide such parameters as: How do you get to work? With whom do you work? What is the setting? How did you like your job? What happens during a typical day? Questions like these used as a guided imagery provided a framework for the person to begin to think creatively and focus on career choice.

\subsection{Intuitive Thinking}

López-González and Limb (2012) note that, as great jazz performers expertly improvise solo passages, they make immediate decisions about which musical phrases to invent and to play, on the spot. They further note that studying the effects of emotion during creation may be another piece to the puzzle of what, how, and why we create. After all, a general social assumption is that emotion guides much of artistic creation. Sawyer (2000) notes a five-hour improvisation of Picasso: In his studio, Picasso is painting free-form, without preconceived image or composition; he is experimenting with colours, forms, and moods. He starts with a figure of a reclining nude - but then loses interest, and the curve of the woman's leg reminds him of a matador's leg as he flies through the air after being gored by a bull - so he paints over the nude and creates an image of a bull and matador. But this leads him to yet another idea; he paints over the bullfight image and begins work on a Mediterranean harbour - with water-skier, bathers in bikinis, and a picturesque hilltop village. The freeform inspiration continues. Five hours later, Picasso stops and declares that he will have to discard the canvas - it has not worked. But the time was not wasted - he has discovered some new ideas, ideas that have emerged from his interaction with the canvas, ideas that he can use in his next painting. Picasso says, "Now that I begin to see where I'm going with it, I'll take a new canvas and start again."

Dayan and Di Benedetto (2011) argue that intuition may unconsciously integrate experience and knowledge of employees into responsive and productive decision-making and, ultimately, into innovative solutions, particularly under rapidly changing, turbulent environmental decisions. The perspective is raised that cognition and intuition are two ends of a continuum and thus mutually exclusive, rather than orthogonal constructs. They formally define intuition as "a non-conscious, holistic processing mode in which judgements are made with no awareness of rules of knowledge used for inference and can feel right despite one's inability to articulate the reason". This definition implies that intuitive decisions are executed rapidly upon the basis of an unconscious reasoning process which may have an affective component such as gut feeling, hunches, and 
"sixth sense". Intuition unconsciously integrates one's attributes of knowledge, intelligence, experience, and respect for the unknown into responsive and productive decision-making and, ultimately, into action.

Raidl and Lubart (2001) argue that a common denominator of intuitive phenomena is a feeling of knowing and "knowing without knowing why". Common usage of the term "intuition" often contrasts it with explicit logical thinking and suggests the existence of three different kinds of intuition. First, one can distinguish "socio-affective intuition," which concerns interpersonal relations and operates typically when seeking to understand a person or a situation. It includes sensitivity to other people's needs and feelings, immediate liking or disliking, and the ability to understand and manage situations involving emotional parameters. This kind of intuition is similar to what Bergson saw as "intellectual sympathy," which connects one person to another. A second type of intuition may be called "applied intuition," which is directed toward solution of a problem or accomplishment of a task. Many famous scientists, painters, or writers report having experienced this kind of intuition. Applied intuition helps people make specific decisions, as, for example, in managerial settings. Finally there are intuitions that do not arise in reaction to socio-affective stimuli or a specific search process. This third type of intuition, labelled "free intuition", involves a feeling of foreboding concerning the future. Winston Churchill was well-known for having such precognitions, as when he once had the feeling that he should sit in the car on the side opposite to where he sat usually, and later a bomb exploded under his usual seat.

Raidl and Lubart (2001) note that Jung was one of the first researchers to formulate a psychological conception of intuition, proposing that it was one of four basic psychic functions. He defined it as an unconscious, internally-oriented mode of perception, as opposed to sensation which he viewed as a conscious, extemally-oriented mode of perception that relies on one's sensory organs (e.g., visual or auditory perception). Westcott conceived of intuition as an inference-making mode of thought (used to make judgements and predictions) that used limited information and tended to yield correct conclusions. Considering that the processes underlying this ability were akin to inductive reasoning, Westcott situated the difference between ordinary induction and intuition in the gap between the available evidence and the intuitive conclusion. $\mathrm{He}$ developed tasks to measure intuition that involved making decisions based on partial information. These tasks seem to rely on the trait of risk taking and speed of closure, an intellectual ability.

Raidl and Lubart (2001) further note the development of research specifically on the processes involved in intuitive thought, over the last decade. Ochse considers that "there is good reason for attributing intuitive thinking to the operation of mental routines, unconsciously triggered by configurations of exogenous and/ or endogenous stimuli". For Rosenblatt and Thickstun, intuition arises when a perceived pattern is unconsciously matched to another formerly perceived one that has been stored in memory. Bowers defined intuition as an implicit perception of coherence: the successive clues to coherence activate relevant mnemonic networks until a certain threshold is reached, upon which the coherence can be consciously identified. For Isenberg, intuition involves synthesizing isolated bits of data into an integrated whole. And Rowan proposed that "the mind organizes experiences, relationships and facts into a new condensed pattern which becomes an intuitive feeling". Policastro defined creative intuition as a "vague, anticipatory perception that orients creative work in a promising direction," a kind of tacit knowledge that influences the search for new ideas and the criteria used to evaluate these ideas.

Torrance (1993) notes a conceptualization of the creative thinking process as taking place in the preconscious system. The preconscious is able to scan experiences and memories, to condense, to join opposites, and to find relations at speeds impossible in the conscious system. The resulting intuitions, however, are not very precise and are subject to the primary-process type of thinking. Creative thinking is described as the process of sensing difficulties, problems, gaps in information, missing elements, something askew; making guesses and formulating hypotheses about these deficiencies; evaluating and testing these guesses and hypotheses; possibly revising and retesting them; and, last, communicating the results.

Duch (2007) argues that the three most important (and most mysterious) faculties of the mind needed for intelligent behaviour are intuition, imagination and creativity. Babies and animals do not reason, making logical inferences, be it crisp or fuzzy, but even birds use intuition, imagination and creativity to solve problems. Computers need to show similar qualities. The word "intuitive" in biology, psychology, mathematics, physics and sociology is treated as a synonym of naïve understanding in these fields. Yet in everyday activity very few people (and certainly no animals) base their decisions on logical analysis of all options. Most cognitive functions, such as understanding of human and animal intentions and emotional states, meaning of words or creative thinking, cannot be reduced to logical operations. Intuition is defined in dictionaries as immediate knowing without the conscious use of reasoning, or cognition without evident rational thought and inference. Deliberate thinking is critical, analytic and reasoning-like, while intuitive thinking is rapid, effortless, and perception-like. From a computational perspective modelling intuition is 
relatively simple. Decisions of neural networks (or other models) that learn from data frequently cannot be justified in terms of logical rules. In some cases logical rules that have similar or even higher predictive power may be extracted from trained neural networks. In other cases judgements based on overall similarity provide better decisions.

Duch (2007) continues that neuro-cognitive models inspired by the putative processes in the brain show that these mysterious features (intuition, imagination and creativity) are a consequence of information processing in complex networks. Intuition is manifested in categorization based on evaluation of similarity, when decision borders are too complex to be reduced to logical rules. It is also manifested in heuristic reasoning based on partial observations, where network activity selects only the path that may lead to the solution, excluding all bad moves. Insight results from reasoning at the higher, non-verbal level of abstraction that comes from involvement of the right hemisphere networks forming large "linguistic receptive fields". Psychologists have noticed that rules and similarity judgements form a continuum, with logical rules (including threshold logic and fuzzy logic rules) applicable in relatively simple cases, while prototype-based rules are applicable in situations when many factors are simultaneously taken into account for similarity judgements. For example, medical doctors may use simple norms based on thresholds for some tests, but in case of emergency they have to make fast intuitive judgements, taking many factors into account. Experience leads to intuition, and it is obviously related to similarity evaluation and memorization of many prototypes. Even for simple benchmark medical data, a single P-rule may offer more accurate explanation than sets of logical rules.

Duch (2007) further notes that intuition is usually invoked in the context of reasoning and decision making. Herbert Simon has claimed that AI has reached the stage where intuition, inspiration and insight could be modelled. Intuition in problem solving has two defining characteristics: 1) the solution has to be reached rapidly, and 2) an explanation why the steps leading to the solution have been selected, can not be given. In various experiments novices and experts solving the same problem were compared, and the use of intuition has been clearly correlated with the ability to evaluate similarity, and with the number of patterns stored in long term memory. Knowledge obtained through implicit learning or derived from partial observations (in contrast to the usual supervised learning situation, when full knowledge is provided) over a long period of time cannot be used directly in explicit reasoning. It is represented in a diffuse, rather weak connections, partially in the right brain hemisphere, and thus cannot be accurately summarized in symbolic form. The brain constantly learns to pay attention to relevant features and remembers many patterns. Even in tasks for which rules of correct actions exist intuitive learning comes before rules are discovered.

Duch (2007) argues that good car drivers may have problems recalling driving rules; they just make correct assumptions and predictions. If the problem admits more than one solution, how likely is it that a student will find all solutions? This should depend on the working memory load, or complexity of the search, needed to find all solutions. In complex situations hierarchical decomposition of the problem is necessary, depending on the questions asked. For example, elements of complex electrical circuits may be decomposed into larger blocks, there is no need to assign values to all variables. People in such cases analyze the graphical structure of connections and nodes representing the problem, starting with the elements mentioned in the problem statement. Duch (2007) notes several software implementations of algorithms for learning from partial observations that quickly find all solutions if many discrete feature values are missing. Problems of this type are somewhere in between pattern recognition and symbolic reasoning problems. Neural networks may be used as heuristics to constrain search processes (a core AI technology) in problem solving. Robots, including autonomous vehicles, need to combine reasoning with pattern recognition in real time. Intuitive evaluation of possible solutions to global goals may help to generate rough plans, and to find optimal patterns for behaviour of a robot.

Duch (2007) states that intuition and insight have some similarities, but the sudden Aha! experience that accompanies solutions of some problems has distinct character. Insight is usually preceded by an impasse, frustration after a period of lack of progress, followed by conviction of the imminence of the solution, frequently after a period of incubation when the problem is set aside. A new way of looking at the problem that leads to the solution is accompanied by a great excitement and understanding. The mild version of an Aha! experience is fairly common during discussions when a difficult concept or a confusing description of some situation is finally grasped. The initial process of searching for the solution reaches dead end, but during the search new features are constructed and stored in the long-term memory. Thereafter the failure control mechanism shifts search to another problem space, and new control structures for this process are created in the short term memory. With additional features of the problem generated in previous runs, the new search has greater chances to succeed. However, this explanation may be applied to typical attempts of solving a problem by using several different strategies, without any Aha! Experience.

Duch (2007) continues that only recently neuroscience has provided a deeper understanding of the 
insight phenomenon. Studies using functional MRI and EEG techniques contrasted insight with analytical problem solving that did not require insight. An increased activity in the right hemisphere anterior superior temporal gyrus (RH-aSTG) has been observed during initial solving efforts and during insights. This area is probably involved in higher-level abstractions that can facilitate indirect associations. About $300 \mathrm{~ms}$ before insights occurred, bursts of gamma activity has been observed. This has been interpreted as making connections across distantly related information during comprehension that allow them to see connections that previously eluded them. A series of fMRI experiments confirms these results. According to this interpretation, initial impasse is due to the inability of the left hemisphere, focused on the problem, to make progress. This deadlock is removed when the less-focused right hemisphere adds relevant information, allowing new associations to be formed. An Aha! experience may result from activation by the pre-existing weak solution in the right hemisphere, suddenly reaching consciousness when the activation of the left hemisphere is decreased. High-activity gamma bursts, observed in the insight experiments, influence the left hemisphere priming larger sub-networks with sufficient strength to form associative connections that link the problem statement through a series of intermediate transitions to the partial or final solution. Such solutions may initially be difficult to justify, therefore the feeling of vague but imminent understanding is generated, replaced by real understanding when all intermediate steps are correctly linked. The solution may be surprising, comprising quite a different idea than initially entertained. Gamma bursts also activate emotions, increasing plasticity of the cortex and facilitating formation of new associations. Emotional reaction should be proportional to the difficulty of forming new associations, therefore grasping a new, difficult concept in a discussion generates only a mild reaction, while solving a difficult problem generates strong emotions, activating the reward system.

Dietrich (2007) states that the prefrontal cortex has three main functions in creativity, namely: 1) gaining consciousness of novel thoughts, which become insights when represented in working memory; 2) recruiting higher cognitive functions in developing the insight, including central executive processing such as the direction of attention, retrieving relevant memories, and considering the appropriateness of the insight to the problem; and 3) implementing the expression of the insight, which involves recruiting task-specific skills, techniques and knowledge. Creative insights can occur in one of two processing modes - spontaneous and deliberate - and either of these can direct operations in one or both of two structures - emotional or cognitive - giving rise to four basic types of creativity. Examples of creative insights which employ the deliberate mode of processing for cognitive structures are those which involve high levels of knowledge and expertise, and the methodical, systematic linking of different pieces of information so as to create an overall pattern such as might occur in scientific breakthroughs like Crick and Watson's decoding of the structure of DNA. Creativity which employs the deliberate mode of processing for emotional structures is also seen as methodical and systematic, and this is applied to affective memories, such as insights achieved during psychotherapy. The third type, insights in which a spontaneous processing mode is applied to cognitive systems, are those in which sudden illumination or "Eureka" experiences are reported, such as in Newton's insights into the nature of gravity when watching an apple falling from a tree, or indeed Archimedes' original Eureka experience when he displaced the water in his own bathtub. Finally, when the spontaneous processing mode is used on emotional structures, the creative insights involve intense emotional experiences, such as in the creation of Picasso's Guernica, or of Stravinsky's The Rite of Spring.

Raidl and Lubart (2001) view intuition as a perceptual process, constructed through a mainly subconscious act of linking disparate elements of information. This intuitive mode of information processing is favoured by an ambiguous or poorly structured context, and taps four kinds of information sources: 1) external stimuli (e.g., a person, a place, a situation, a sound etc.) which often trigger an intuition; 2) memory (sensory, episodic, semantic...), in particular implicit memory, that is, all the experience acquired over time, routines, or know-how, 3) emotions, which constitute a vast reservoir of information; and 4) subconscious concerns, which result in the activation of certain representations and can have a priming effect. Intuition is shaped out of the connection between some of these elements. It may be that only one element serves to initiate an intuitive feeling, for example, a strong emotion, not explicitly appearing in reaction to an external stimulus. Usually, however, several elements interact in order to produce an intuition. The fact that intuitions seem often to appear without a clear reason is due to the subconscious nature of the associative processes and to the fact that they allow very distant content areas to be put together. Two points are important in the underlying process of information combination. First, encodings need to be rich. An intuitive person is one who perceives and stores many environmental cues which may be subtle and non-explicit (e.g. non-verbal communication). Second, concerning the combination of diverse elements of information, several processes may come into play. For example, inductive reasoning and logical extrapolation from known data seem involved together with more subjective processes such as empathy, projection, or emotional resonance. Finally, associative processes such as metaphorical and analogical thinking may be at work. Kolanczyk has stated the importance of metaphors in 
intuition: "one of the most effective ways of activating intuitions is through metaphor... (it) associates concepts with episodes and sensory experience. This is probably a side door into the search of meaning formation in intuition".

Harvey and Novicevic (2002) note that it has been theorized that expatriates who have a heightened intuitive intelligence (that is, the part of an individual's "intelligence" which concentrates on the possibilities, less concerned with details or facts, and finds solutions directly without necessarily basing decisions on known facts - the ability to gain quick insights into how to make decisions, frequently referred to as "street smarts") will be more successful. The organizational benefits of using the intuition of expatriate managers during their overseas assignments are: 1) expedited decision making process; 2) a qualitative improvement of decisions by relying on experiential base of informal knowledge that is not generic to the organization; 3) facilitated personal development by building personal self-efficacy of the expatriate decision-maker (having insights and confidence that others do not have in making decisions in complex environments); and 4) promoting decision compatibility with the headquarters goals and mission due to the shared experiential insights into these issue. The intuitive expatriates can provide specific experiential insights relevant for the interrelated organizational processes or routines. These insights are rooted in the expatriate's intuition, given past experience and success in making accepted decisions in the organization. Coupled with the concomitant social capital that the expatriate has built over time in the organization's headquarters, it is logical to conclude that the expatriates will have a high self-efficacy, thereby enhancing their ability to use their intuition to make future decisions. In many ways, intuition suspends the bounds of rationality in unstable and complex environments like hypercompetitive markets to permit the expatriate decision-maker to intuitively frame particularly complex problems that they have not faced before and at the same time propose potential effective solutions. Expatriate managers have to trust their judgements and overcome their fear to use intuition, in that, it is not merely an emotional reaction to complexity, but rather can be an evolved means to estimate how to address "unknowns" in the environmental framework. The most important element of using expatriate intuition is the speed with which decisions can be made, and recognizing that intuition is critical for agility in hyper-competitive situations.

Harvey and Novicevic (2002) continue that there is evidence that supports the contention that tacit knowledge of an operating environment stimulates the use of intuition and provides the incentive for an expatriate to be a creative action-oriented decision maker. When expatriate managers are faced with complex unfamiliar decisions, but yet have tacit knowledge of how the organization works (as well as, what is the acceptable level of risk taking), they can successfully improvise in their decision-making processes. Managerial improvisation is the enactment of action as it unfolds, by drawing on available material, cognitive, affective and social resources. Improvisation has a deliberate intentional element as well as an extemporaneous unplanned dimension that are based on "acting on the moment". Improvisation is a critical concept relative to expatriate intuitive intelligence in that it provides foresight for decision making in unexpected situations, or when the dynamics of the environment does not allow for a preplanned course of action. In these situations, the intuitive intelligence activates the expatriate manager's ability to use improvisation to develop strategies on the "run". This ability to reflect and act simultaneously is frequently referred to as an evolved "gut knowledge" that differentiates excellent from average expatriate managers. Intuitive intelligence becomes the enabling mechanism for expatriate managers to improvise when the complexity of the environmental change is very high, and there is limited past experience for making decisions. One of the key considerations in assessing the creative intelligence of expatriates is to examine their ability to recognize patterns and/ or to be able categorize people, events, environments into classification schemes of opportunity. Patterns are also useful when attempting to integrate two systems or organizations (home and host country). By recognizing similar patterns between two entities in different environments, the expatriate manager is able to determine what aspects do not need to be relearned or modified between the foreign and the home environments. The ability to categorize problems into classification schemata learned in their domestic experience enables expatriates to pay selective attention to exceptions or problems in the hyper-competitive global marketplace that need creative solutions.

\subsection{Learning}

Siegler (2006) notes that the relation between learning and development (short-term and long-term change; micro-development and macro-development) is among the enduring issues in developmental psychology. Some theorists view the two as similar. Others see them as fundamentally dissimilar. One view is that development creates new cognitive structures; learning merely fill in specific content.

Nuangchalerm and Thammasena (2009) note that, in science education, inquiry-based learning allows students to learn by doing, and students learn how to solve problems by themselves. This method is a complex, 
but realistic process in which students use their prior knowledge and scientific theories to generate new understandings of science. The idea of teaching science by inquiry method encourage students to explore and experiment with their own concepts of science. In addition, inquiry-based learning can be related to the diverse ways in which scientists study the natural world and propose explanations based on evidence derived from their work. It includes the activities of students in which they develop knowledge and understanding of scientific ideas, as well as an understanding of how scientists study the natural world.

Hargadon and Bechky (2006) note that, within the organizations they studied, individuals chose social interactions that retained the equivocality of past experiences - the multiple meanings that might be considered and reapplied in a new context. As one Hewlett-Packard engineer described: When you read about somebody's experience and then actually go and talk to them about it you find the level of knowledge is so much deeper than what can be transferred through a paper or an hour-long talk. There's a wealth of hidden knowledge that's a result of the struggles, the agonizing they went through to try to figure out what's the right way to proceed rather than the wrong way.

Kolb et al. (2001) argue that experiential learning theory (ELT) is distinct from both cognitive learning theories, which tend to emphasize cognition over affect, and behavioural learning theories that deny any role for subjective experience in the learning process. Experiential learning theory defines learning as the process whereby knowledge is created through the transformation of experience. Knowledge results from the combination of grasping and transforming experience. The ELT model portrays two dialectically related modes of grasping experience - Concrete Experience (CE) and Abstract Conceptualization (AC) - and two dialectically related modes of transforming experience - Reflective Observation (RO) and Active Experimentation (AE). Immediate or concrete experiences are the basis for observations and reflections. These reflections are assimilated and distilled into abstract concepts from which new implications for action can be drawn. These implications can be actively tested and serve as guides in creating new experiences. In grasping experience some perceive new information through experiencing the concrete, tangible, felt qualities of the world, relying on the senses and immersing themselves in concrete reality. Others tend to perceive, grasp, or take hold of new information through symbolic representation or abstract conceptualization - thinking about, analyzing, or systematically planning, rather than using sensation as a guide. Similarly, in transforming or processing experience some tend to carefully watch others who are involved in the experience and reflect on what happens, while others choose to jump right in and start doing things. The watchers favour reflective observation, while the doers favour active experimentation.

Kolb et al. (2001) continue that ELT follows Carl Jung in recognizing that learning styles result from individuals' preferred ways for adapting in the world. There are four prevalent learning styles - Diverging, Assimilating, Converging, and Accommodating. The Diverging style's dominant learning abilities are Concrete Experience (CE) and Reflective Observation (RO). People with this learning style are best at viewing concrete situations from many different points of view. People with a Diverging learning style have broad cultural interests and like to gather information. Research shows that they are interested in people, tend to be imaginative and emotional, have broad cultural interests, and tend to specialize in the arts. In formal learning situations, people with the Diverging style prefer to work in groups, listening with an open mind and receiving personalized feedback. The Assimilating style's dominant learning abilities are Abstract Conceptualization (AC) and Reflective Observation (RO). People with this learning style are best at understanding a wide range of information and putting into concise, logical form. Individuals with an Assimilating style are less focused on people and more interested in ideas and abstract concepts. Generally, people with this style find it more important that a theory have logical soundness than practical value. The Assimilating learning style is important for effectiveness in information and science careers. In formal learning situations, people with this style prefer readings, lectures, exploring analytical models, and having time to think things through.

Kolb et al. (2001) expand that the Converging style's dominant learning abilities are Abstract Conceptualization (AC) and Active Experimentation (AE). People with this learning style are best at finding practical uses for ideas and theories. They have the ability to solve problems and make decisions based on finding solutions to questions or problems. Individuals with a Converging learning style prefer to deal with technical tasks and problems rather than with social issues and interpersonal issues. These learning skills are important for effectiveness in specialist and technology careers. In formal learning situations, people with this style prefer to experiment with new ideas, simulations, laboratory assignments, and practical applications. The Accommodating style's dominant learning abilities are Concrete Experience (CE) and Active Experimentation (AE). People with this learning style have the ability to learn from primarily "hand-on" experience. They enjoy carrying out plans and involving themselves in new and challenging experiences. Their tendency may be to act on "gut" feelings rather than on logical analysis. In solving problems, individuals with an Accommodating learning style rely more heavily on people for information than on their own technical analysis. This learning 
style is important for effectiveness in action-oriented careers such as marketing or sales. In formal learning situations, people with the Accommodating learning style prefer to work with others to get assignments done, to set goals, to do field work, and to test out different approaches to completing a project.

Kolb et al. (2001) state that integrated learning is conceptualized as an idealized learning cycle or spiral where the learner "touches all the bases" - experiencing, reflecting, thinking, and acting - in a recursive process that is responsive to the learning situation and what is being learned. Adaptive flexibility in learning refers to the degree to which people change learning style to respond to different learning situations in their life. Mainemelis et al. (2002) continue that this development in learning sophistication and creative adaptation results from the integration of the dual dialectics of conceptualizing/ experiencing and acting/ reflecting. Adaptive flexibility is related to the degree that one integrates the dual dialectics of the learning process conceptualizing/ experiencing and acting/ reflecting. Both the conceptualizing/ experiencing dialectic (naming one's own experience in dialogue with others) and the transformative dialectic between reflection and action (reflection informed by action and action informed by reflection) carry importance.

Lumpkin and Lichtenstein (2005) note three themes of organizational learning: behavioural learning, cognitive learning, and action learning. Behavioural learning sees organizations as goal-oriented, routine-based systems which respond to experience by repeating behaviours that have been successful and avoiding those that are not. Trial-and-error learning leads to routines and processes that confer selective advantage to the firm. Emphasis is on learning from repeated behaviours. Cognitive learning focuses on the cognitive content of organizational learning and how changes in individual's cognitive maps are aggregated and translated into changes in an organization's cognitive schema. The focus is on the content of learning rather than on its behavioural outcomes, on processes that improve the creation of knowledge in a firm, and the utilization of knowledge to improve creativity, quality of interaction, and other types of performance. By putting the right processes in place, a learning organization can, in essence, transform data into information, and information into knowledge, which can then be leveraged to generate organizational knowledge. Organizational learning, in this sense, includes the process of exploiting externally-generated knowledge or transforming internallystored knowledge.

Lumpkin and Lichtenstein (2005) continue that action learning focuses on the moment-to-moment practice of correcting misalignments between "espoused theory" (what individuals or organization say they do) and its "theory-in-use" (what individuals or organizations actually do), to produce more effective action in real time. Action learning is primarily concerned with the patterns of belief and qualities of interaction between organizational members that facilitate (or constrain) the capabilities of the firm. Such learning is simultaneously personal and organizational, as it is built through a commitment to improve the integrity of individual action, as well as the alignment of activities within the organization. When a group of individuals commit to an action-learning approach, a community of learning practice can be generated that may significantly impact the quality of communication, innovation, and team performance in a firm. According to this approach, learning happens in "real time," through a nearly simultaneous reframing of personal belief and action that can transform the individual as well as the organization.

\subsection{Composite Thinking}

Webster (2002) notes that creative thinking is a dynamic process of alternation between convergent and divergent thinking, moving in stages over time, enabled by certain skills (both innate and learned), and by certain conditions, all resulting in a final product.

George (2007) notes how unconscious thinking contributes to creativity. Imagine a developer is trying to come up with an initial prototype for a new concept and line of dishwashers. While consciously working on this task, the developer identifies a number of features of form and function and options for the line that seem viable but not terribly exciting or unique. Not under a pressing deadline, the developer decides to put the project aside for a while and complete other tasks with deadlines. While putting together an unrelated PowerPoint presentation a few days later, an idea for the prototype that combines elements of the previously identified options in a unique manner pops into his mind. In each of these cases and countless examples from personal experience, conscious and unconscious thought work together.

Duch (2007), López-González and Limb (2012) and Abraham et al. (2012) all note the diverse number of brain regions involved in creative thinking, the higher cognitive functions that take place in the brain, and also the progression of active brain regions during creative thinking. López-González and Limb (2012) state that, even with relatively few completed studies, researchers have concluded that musical creativity clearly cannot be tied to just one brain area or process. They note that creative behaviour is ultimately the result of a combination of the four psychological processes: deliberate cognitive, deliberate emotional, spontaneous cognitive, spontaneous emotional. 
Duch (2007) argues that words in the brain are an abstraction of acoustic speech input, changed into phonological, categorical representation. Categorical auditory perception enables understanding of a speakerindependent speech and is more reliable in a noisy environment. Phonemes, quantized building blocks of phonological representations (typically about 30-50 in most languages) are linked together in ordered strings by resonant states that represent word forms. In brains of people who can read and write, strictly unimodal visual representation of words in the Visual Word Form Area (VWFA) in the left occipitotemporal sulcus has been found. The adjacent lateral inferotemporal multimodal area (LIMA) reacts to both auditory and visual stimulation and has cross-modal phonemic and lexical links. It is quite likely that the homolog of the VWFA in the auditory stream is located in the left anterior superior temporal sulcus; this area shows reduced activity in developmental dyslexics. In the Broca's area in the frontal lobe precise motor representations that generate speech are stored. All these representations of word forms help to focus thinking processes. Activations of word forms are correlated with activity of other brain circuits, pointing to some experiences, perceptions and actions that define the meaning of words. Polysemic words probably have a single phonological representation and differ only by semantic extension. Analysis of the N200 feature of auditory event-related potentials shows that phonological processing precedes semantic activations by about $90 \mathrm{~ms}$. Similar phonological word forms activate adjacent resonant micro-circuits. To recognize a word in a conscious way, activity of its sub-network must win a competition for an access to the working memory. Hearing a word activates strings of phonemes, priming (increasing the activity) all candidate words and non-word combinations. Context priming selects extended sub-network corresponding to a unique word meaning, while competition and inhibition in the winner-takes-all processes leaves only the most active candidate network. Semantic and phonological similarities between words should lead to similar patterns of brain activations for these words.

von der Malsburg (1997) argues about the unity or coherence of introspective experience, by focusing on how consciousness switches between mental states and modalities. From the causal criterion for the state of consciousness, it is clear that a snapshot of a brain or of a mind, taken with a super-cerebroscope, cannot tell at all whether that organism was in a conscious state: causal tests could not be performed. The method for doing so is to create events that could not possibly have been predicted (in their timing, for instance) and to test whether they have consistent consequences. Thus, consciousness cannot be conceived as a static configuration, but only as a historic process in time. This then leads to another Gedanken-experiment: Use the super-cerebroscope to record the brain process over a whole interval of time and store the result on a magnetic tape: Would that tape (or a "slave brain" controlled in detail by it) be conscious? To make the question more pointed, assume the recorded period contained clear examples of events that evidently were unexpected by the brain, and the record showed that each and every time all the different agents had reacted appropriately. The inclination would be to say that the recorded brain process was a conscious one, but that nevertheless the tape itself wasn't conscious because it certainly could not react to stimuli thrown at it: the replay of the tape would be a logical, not a historical process.

von der Malsburg (1997) continues that sub-conscious states can be interpreted as those in which many of the agents in the brain are not functional or are not coherent with each other. Sub-conscious activity may go on in the mind while we are consciously engaged in another matter. We usually find out about such activity in indirect ways, by discovering effects that cannot be attributed to mind processes that we remember, or if we remember them, cannot be traced back to a reason or purpose or to an act of will. The basis for classifying mind processes as sub-conscious is the judgement that one or several important modalities have not contributed properly to the process. Apparently, the lack of functionality of modalities, or their lack of coordination, doesn't correspond to sheer lack of neural activity. In fact, electro-encephalograms and other signals recorded from the healthy brain suggest that the whole brain is always active. The lack of mutual engagement of modalities or the total absence of some of them as active agents in the mind is probably rather due to the lack of correspondence or coordination or resonance, and therefore proper interaction, between sub-processes. A completely unconscious state of mind, in this image, is one in which there is a very low level of coherence between sub-systems, to the extent that one cannot talk of a functional state at all, the brain not capable of reacting to any event altogether.

von der Malsburg (1997) further argues that a thought can establish itself in the mind only if none of the participating modalities throws in its veto. In a fully conscious state this is a very restrictive condition. Since among the modalities there are some that tell us how similar situations have been handled in the past, the conscious state has a tendency to restrict us to trodden paths. Disengaging modalities from a process liberates it from constraints and gives it more freedom to be creative (but also to err, of course). Working at a problem intensely and consciously (and bearing the frustration of not being able to solve it right away), and then doing something else to engage the conscious mind in other activities and to let the sub-conscious silently do its work: when returning to the problem, it may be found that all of a sudden the pieces fall into place and 
the solution is seen in a flash. This account easily jibes with the coherence definition of consciousness: While many of the usual modalities are out, being engaged in everyday activities, some idle agents are on their own, engage in play among themselves in ways that would never be permitted by some others, and before those come back they have found and locked in patterns that are novel and crucial to the solution sought. When my finger touches an object in front of me, my eye is able with great precision to predict the moment when my sense of touch will record the actual contact. This agreement can only be reached reliably if the participating modalities, vision, kinesthesia and touch, separately have a correct representation of the actual situation. Similarly, translation of Chinese into English (taken as two modalities) is not possible as a logical process without reference to a representation of the subject matter spoken about. Representation and meaning (Mephisto's "Schein des Himmelslichts" in Goethe's Faust), so much taken as the very essence of consciousness, would therefore be a functional must and not a superfluous luxury.

Sutton and Hargadon (1996) note that brainstorms help IDEO designers acquire, store, retrieve, adapt, and combine knowledge of potential solutions to design products. McFadzean (1998a) argues that one method of encouraging creativity is to bring together teams of people so that participants can spark off new ideas from other group members as suggestions are made. Top quality ideas have also been shown to occur when groups use a group support system that allows the participants to remain anonymous. Creativity is enhanced when experience, ideas and diverse elements are mixed together and then transformed using new combinations. This is known as association and is the basis of many creative problem solving techniques.

Hender et al. (2001) note that many organizations are recognizing the importance of teams for problem solving, because the necessary information is often distributed among group members, and cooperative problem solving by a group may lead to acceptance at the implementation stage. However, getting a group of experts to work together efficiently and effectively can be difficult. Group Support Systems (GSS) can mitigate some of these problems, by providing at least three functions: parallel communications, anonymity, and group memory. Parallel communications can help a group during idea generation by allowing individuals to communicate simultaneously, thereby reducing production blocking, whereas anonymity helps reduce evaluation apprehension. Both of these result in more equal participation. Group memory supports creativity by allowing participants to view ideas generated by others and hence facilitates piggybacking of ideas.

Similarly, Garfield et al. (2001) note that, in forming idea factories, most companies have focused on finding creative people and giving them the resources they need, because there is a long history that shows that some people are simply more creative than others. However, the exposure to ideas from other team members and the use of creative problem-solving techniques may be at least as important in creative idea generation. While idea generation can be an individual activity performed in isolation, in most cases people do not generate ideas in isolation; often they work with others, as part of a formal or informal group to generate ideas. The contributions of other individuals who are engaged in similar idea-generation activities affect both the ideas produced and the subset of ideas actually contributed. From a cognitive perspective, it is the external stimuli the ideas from others - that trigger one's own cognitive activities.

\section{Methodology}

In order to further examine the creative process of innovators and entrepreneurs, semi-structured interviews were conducted with 10 participants. Purposive sampling was used to select the participants. The participants were mostly established entrepreneurs, all having extensive experience (all participants far exceeded the study's minimum of at least 3 years), and owning at least 1 business. The interviewees have been directly involved with innovation and entrepreneurship, with appropriate level of experience. It was not considered necessary to constrain participant selection according to industry type, and the participants cover a number of industries. The participants were asked 29 questions, and the interviews on average lasted 1 hour. The interviews were recorded, transcribed and subsequently coded.

\section{Analysis}

\subsection{Attributes of Creativity}

Creativity implies an ability to re-imagine the problem. It requires colourful thinking, and deeper understanding or thinking. One needs to look beyond boundaries and the obvious. The objective is to start something new, or something not done before: something new, an improved version of something, or something done in a different way. It is about seeing things differently, as a way of transcending to the next level. Therefore, to objective is to not compete on the same points, and to differentiate yourself and your work.

Creativity is about perspective. It concerns the way you look at things, It is the ability to see 
opportunity from a different perspective. Seeing what is not there, and looking at the problem from a strategic, holistic, and detached perspective. It is about having the proper frame of mind. Seeing behind the curtains, and seeing the complete picture. Instead of looking at something as it is, it is looked at in terms of its potential what it could be. It involves free thinking, lateral thinking, pattern making and dot connecting.

Creativity is based on insight. It is the ability to fundamentally understand the problem, system or game, and the dynamics thereof, so that you are able to work in on it.

Creative thinking involves meta-thinking. It is not just putting the idea together, but also better understanding of the depth of the problem, or the next level of what you are trying to solve. It involves the ability to work at a high level in multiple, parallel fields.

Creativity incorporates focus. One can typically not fully understand complex problems. Thus, rather focusing and finding aspects of the problem to solve. In turn, focusing on the right part of the problem - the most relevant part.

Creativity can mean to break from the ordinary or conventional. It requires thinking outside of the box. To think of uncommon things or ideas, to start with concepts that do not necessarily make sense, or to pull the carpet out among things.

In turn, creativity is nourished. It is founded and has an environment and culture. Its environment can be compared with a garden - one need to create a garden (environment/ culture) in which creativity can flourish. Creativity inspires and changes culture, and in turn is affected by culture. Language as a reflection on mindset or orientation, has a key role. For example, not mentioning or talking of mistakes, but rather speaking of learning. Thinking and learning to think are encouraged, thinking is not classified, and constraints are not placed on thinking. Freedom is given to try things to see if it works. Creativity can become habitual or second nature - a daily habit; to not just wait until things demand change, and so forth, but to look at things all the time from a creative perspective. The innovator is always looking for new ideas, and is always looking for new channels. Creativity may be likened to stepping into a creative realm.

Creativity may be linked to and may build on intelligence or cognitive development. Currently, there is an assimilation of, or ease of access to, information. It means everybody else pretty much has access to the same information or resources - the same status quo or base line. Thus, in order to solve a problem one has to look well beyond what approach most people might take to solve the same problem. This implies looking beyond merely solving the problem, but also noting how else the solution can be enriched, to make the solution far more attractive. It implies optimizing the solution.

Creativity is about constructive thought, synthesis and stimulation: looking for things in the normal, from everyday life, and picking it out to build on. The innovator is constantly making connections and matching patterns. For example, he will hear somebody mention a comment, and he will think of a book. It is not just a single piece of information or inspiration that creates the creative thought, it is combining a number of things into a whole more powerful than the sum of the parts.

Creativity is contextual. It requires a problem or context to commence from. Creativity is proactive. It implies thinking, instead of merely reacting. Creativity is purposeful and practical. It introduces change, and at the same time value. It requires being practical and getting the practical as well as business side of creativity right. It incorporates market intelligence, and it equally understands the dynamics of the market. True creativity merges the left and right brain.

Creativity requires sound judgement. It considers the views and opinions of others, but also understands their perceptions. It knows how to process opinion, and when people's opinions are irrelevant. It knows how to align with opinions and perspectives - when to go with, and when to change the perspective. It knows when to follow the market, and when to rather craft or shift the market. There generally lays greater creativity in crafting the market.

Creativity is not confined to rational - or even linear - thinking only, it is a lot more about shattering boundaries and thinking. It permits more openness in terms of ideas, and it does not judge ideas beforehand or a priori, based on personal thinking and mindsets. Creativity my leverage intuition, and may spot opportunities intuitively. Whilst in the middle of something, a quick opportunity that is right for the time may be recognized.

Creativity is less about boundaries, and more about transcending boundaries. The innovator have to be a bit of a dreamer, in order to look at something differently.

Creativity believes. It believes that something else can exist, and this allows divergent thinking. It is thus able to overcome and transcend perspectives based on stereotypical thought.

Creativity has elements of anticipation, proactiveness and resourcefulness. It takes a proactive stance and is resourceful in terms of solutions. It is able to look into the future and anticipate what is going to be relatively more and less important, with regards to trends.

Creativity pays attention to detail, and is aware of the surrounding environment, and the innovator 
recognizes what is around him. He realizes the need around him.

Creativity may be depicted as the opposite of psychological inertia. Psychological inertia refers to opinion and brain patterns that we have established in our lives through upbringing, etc.

Creativity is equally about acting a bit insane: doing the same thing and expecting a different result, and being persistent: keep on (at) failing, while continuing to believe that there is a way out - a solution - and that it is merely a case of having to change the steps in between.

Creativity is expensive. A myth about creativity may be that it is like a spark of inspiration. Related to this is the misconception of the artist and the muse - that great ideas strikes suddenly and you are set. Creativity is grounded. It involves a context and a lot of (different ways of) thinking.

Attitude may be what differentiates the innovator, in that attitude determines how much the innovator learns from experience, and thus develops creatively. Instead of playing victim or blame shifting, he may actually endeavour to learn from experiences, and in doing so develop his own experience and intuition. Rich experiences make creativity and finding solutions easier. The true innovator also puts in effort to reflect on his experiences and creative attempts, to again learn from it and develop through it.

\subsection{Creativity Traits}

The amount of creativity a candidate possesses over must be experienced; it is not on the surface. Some of the things that demonstrate and reveal how much creativity a candidate possess are: 1) how intuitive they are, 2) how much foresight they have, 3) how proactive they are, 4) how much initiative they show, 5) how open a person is to suggestion, 6) how willing a person is to explore.

How a person thinks and what he talks about are important. He is generally inquisitive and his interests, stories, ideas, experiences (implementation of ideas) that he articulates, speak of itself. The degree of abstract thinking and conceptual understanding he demonstrates. He should show high levels of interest and curiosity - an absolute desire to know as much as possible. The candidate should prove himself to be open minded - not thinking in terms of lines or boundaries or categories too much - not putting things in boxes, or able to change or redefine the boundaries or categories.

The candidate should be able to deal with ambiguity: he must be able to uncomfortable with something - to only have a loose definition or conceptualization of something, and to perhaps have more questions than answers regarding something - for a period of time, without knowing how long it may take for a sense of stability or order to return.

His mental predisposition, and his behaviour may also be telling. The way the person dresses, walks, carries himself, uses his hands, and converses, may all reveal his creativity. People that are stronger right brain, or people that are mentally more balanced, tend to be more creative, and mental predisposition quickly becomes apparent. A creative person generally likes to talk longer, frequently changes his tone of voice, and carries a lot of excitement. Creative people also demonstrate greater levels of self-determination.

It is very easy to pick up whether a person has original thought or a copy cat idea - it is soon apparent whether a person thought through something. The view is also that education plays a role to some extent.

Simple observation can reveal creativity via things like problem solving. For instance, "you can look at a kid in a sandbox, or in a playschool, and you would be able to see the creative ones. You would be able to see the ones that are solving the problems by innovation ideas, and the others just become a victim to the problem."

Simple tests - for example, what someone can do with a paper clip - and intuition built up through experience, also help to assess a person's creative capabilities.

\subsection{Creativity as Skill}

Some of the significant points that surfaced through the interviews are: 1) the ability to develop creativity through practice, and 2) the role that upbringing plays.

To many, they felt that creativity came through their intuition - they have an intuition for creativity and it was within their natural abilities - they are naturals at creativity, and creativity is a natural state of mind to them.

The general view is that anybody can be creative. Even if not every person is born with creativity capabilities, it is still a skill that can be learned through proper education. The brain is seen as a key contributor to or medium of creativity, and like any other part of the body, the more you exercise it, the better or smarter it becomes. Much of creativity entails becoming a better thinker, and learning to think creatively is seen as a skill that needs to be honed. It requires confidence to be creative, and people should generally be helped past the first step. Creativity improves with practice, and requires time and room to act on curiosity. When placed in the right environment, anyone can and will be creative. The brain needs to be exercised to think creatively, 
and this must be done often and regularly. In this regard, the view is that education and upbringing do not enough.

Children are all predominantly creative, they are further conditionalized and shaped by rolemodels, like their parents, and their upbringing though, and unconducive environments equally have an effect. Similarly, creativity can be developed and improved. Being exposed to creativity and creativity examples/ practice can help in this regard. For example, watching TV (documentaries that reflect on creativity, etc) may feed the sub-conscious and stimulate and encourage creativity this way.

Upbringing frequently surfaced during the interviews. A number of participants recalled their youth, and being creative during/ since their youth already. They demonstrated the potential to be creative since childhood, and have had a creative side. They played in creative ways, and were inventing things - were innovative - during childhood. They were imagining and visualizing things. And they enjoyed doing it. Also, they have received help and encouragement, notably from key roleplayers like their parents, and their creativity was allowed to develop. The urge to be creative is not really stronger now than then. One participant had to improvise a lot as a child. His parents were not rich, and he had to make what he wanted.

In many cases, the participants felt that their understanding of creativity, rather than creativity itself, developed with time. They have learned to harness, channel, focus, control and use it more efficiently, and to be more disciplined about it. They build experience, allowing them to be more creative. At the same time, if creativity is only defined as creative - or free - thinking, some see people many years younger than them, and thus with less experience, just as creative as they.

One participant in particular demonstrated the wilful effort to be creative. The creative side did not perhaps come natural, but the person put in deliberate effort to develop it. The person sought balance between the left and right brain, and actively got involved in creativity - becoming part of the process of designing things and making things (in his case, through investing in companies).

Routine is seen as a significant impediment to creative thinking.

In support of the point that creativity is equally developed, innovators (participants) will typically mention that they initially work with single models, viewing the problem from the perspective of a number of single models. After having familiarized themselves with individual models, they subsequently start to use multiple models, both in combination and simultaneously. They also start to more frequently and fluently shift gears - changing between the models and tools at their disposal. They become more experienced and fluent in the overall creative process, such that they move through the process faster, the more it becomes second nature, and they no longer consciously think about it.

\subsection{Stimulants}

The innovator generally knows when he is being (exceptionally) creative, and may sense something happening in his brain. He may need to do different things to access or achieve that state. It can be haphazard - when he is alone with a piece of paper, or when he is facilitating a discussion or workshop.

Some of the common stimulants of creativity are: 1) being inquisitive and seeking to truly understand things, 2) collecting a lot of general knowledge, 3) getting and stepping out of the traps brought about by personal successes, 4) mental stimulation like music (and narcotics), emotions (emotional experiences), or real-life experiences (beauty (nature), the unconventional and abnormal, triggers, interrupts, events, occurrences, experiences, situations), 5) visualization and dreaming, 6) desperation, necessity and pressure, 6) being fascinated by a problem, being passionate about something, or being vested in something, 7) simply enjoying the challenge, being creative, and doing creative work, 8) a change in the environment, and 9) specific times or events.

Creativity is also sparked by problem solving. Being confronted with a problem often instigates the desire to find a solution. This is also in line with human nature. When faced with a problem, some may attempt to bypass or ignore it, whilst others will attempt to propose a solution. Even if it is not the best idea, they will come up with an innovative idea to address the problem. Thus, to be open to the problems people are experiencing can stimulate creative thought.

Some are of the opinion that desperation, necessity and pressure, including fear and stress - when things are tough and when your back is up against the wall - may equally instigate creative thinking, whilst other confess the opposite - they require peace and quiet, with no stress. The person may require space, time and quietness - the ability to think and concentrate without interruption - to be creative. When under pressure, the person may switch from panic mode to a recovery and salvage - pioneering - mode. He may simply identify and accept the challenge he is confronted with, and see it as such.

At the same time, creativity is also seen as strategic or planned. Creativity or creative thinking can be (made) systematic, with routine to it, and tools to aid creative thinking. Creativity can become second nature 
and routine.

The effect a change in the environment can have on creativity and creative thinking was mentioned a number of times. Related to this is the national culture. The right environment will allow the brain to be most creative, for example, people may experience "monkey brain" (only thinking in terms of 3 actions: freeze, flight, fight) when under pressure. The right environment implies that it is in order to be stupid and ask stupid questions, to completely step outside of the norm. At times, a change in the environment or perspective may be deliberate, to stimulate creativity. For example, being confronted with or exposed to something that is totally contrary to the current situation, and deliberately putting oneself in a different context or realm, to see the world differently. When creativity is practised in a team, attention should be paid to putting together the team and improving its dynamics.

Interaction with people, and being among certain people and the right people can also stimulate and boost creativity. For example, being around people with like minds and passions, or to be in a group that goes overboard to a degree - that demonstrates freedom and spontaneity. Or, interaction with other people that helps one to build on knowledge and to exchange ideas. Exchanging ideas with potential customers can give further direction to creative thinking.

The mind itself may be an important contributor to creativity. Examples would be the ability to explore ideas within certain confines, having a curious mind, or having a mind that is open to and able to consider a number of things or points at the same time, and to then be able to integrate it. The right mindsets can also enable creativity. An example would be to train yourself and making a commitment to see the world differently. It is also important to clearing the brain. The brain gets full or occupied, and it is beneficial to clear and reboot it when moving from one task to the other. In addition, the mind may also be warmed up to start thinking creatively through thought exercises.

Dreams or visions of the future or what may be, may put creative thinking into motion. The actual benefits of being an entrepreneur can serve as motivation as well - enjoying greater autonomy and freedom. Some express that they are particularly more creative during particular days or particular times during the day.

Creativity is also seen as an way to escape the norm. It is seen as a necessity, if one desires more, and a different life or outcome than the norm. Creativity may be the result of not wanting to settle in routine, and personal aspirations, self-actualization, and the need for greater stimulation may lead to creativity.

Creativity may simply be the consequence of being employed in a profession and area that generally require and allow creativity. This would also dictate the way the person thinks - the person may be taught to think creatively. A number of participants expressed having an artistic side - being involved in arts, or (writing) music.

\subsection{Process}

\subsubsection{Formal Process}

The overall view is that there is no formal, straight-forward or cut and dry process to creativity. At the same time, there may be a standard procedure or routine to creativity. The innovator as creative person has a general routine, procedure or outline he normally follows during the creative process, that is rather standard. There are a number of distinct modules or processes that his thinking progresses through or along. He will generally contextualize the problem to be solved, form a rough idea how to solve the problem, and then work to refine the solution. He may reference and search for models, frameworks and tools to build perspective around the problem and its solution. The experience he has built up over time may prove invaluable, and he may draw extensively from it. Also, he may commit to a quick feasibility study, looking at things like cost and the market, and may speak to people close to him or in his network to form and expand a quick opinion of the problem and solution. At that point, he will start to focus on the details of the problem or solution. Certain information can not be gathered from background research, people or his network. In these cases it helps to get closer to the problem, and to experience the problem first hand. During the process, there are a number of things he will consider and incorporate, and he may utilize a number of models and tools to this effect. For example, he will look at ways to differentiate away from the competitor, turn the industry forces into gains, and differentiate the value proposition in terms of the customer. He will conduct significant research to validate and test his conceptualization, premises and assumptions regarding the proposed solution.

Creativity is partially structured and partially unstructured. No two problems or solutions are ever the same and creativity also requires flexibility. Many times, the solution may not be obvious. The innovator may not get that far and may get bogged down when trying to define and articulate the problem, and may only move forward when allowing some room for ideas to linger and gestate. That is, for creativity to happen and for original thought to occur: creativity involves a lot of original thought that does not progress in a linear or structural manner. Again, the confines, moulds or processes of creativity may be well-defined, but its flow is 
not necessarily well-defined.

There is a part of creativity or the creative process that is more work than thinking, and hard work that is. The example is given of an artist that has to paint for hours and hours. A lot of this pertains to implementing the creative idea or thought.

\subsubsection{Evolution of the Solution}

What the innovator thinks is the answer, and what turns out to be the answer, may be far apart. His perceptions of the problem, and the realities of the problem may differ. He may very well discover what he thought the problem was, is not really the problem. Many times, how he sees a problem is not really what the problem is, and there may be layers to the problem. The problem may be more complex than meets the eye. The real problem is either something deeper or closer to the surface, or a completely different problem. Initial perceptions are likely to change or evolve, as the innovator slowly learns the true dynamics of the problem, and as he further experiences the problem. It is only possible to pick up certain properties and intricacies when the innovator is in the system, and immersed in the problem, and the real issues of the problem may only surface whilst working on the problem. More knowledge of the problem may better reveal the most important issues, and may change the direction or solution taken.

It is not possible to grasp all the dynamics beforehand. Like strategy, products evolve. Doors open and close - opportunities, options and possibilities come and go. It is not possible to anticipate everything, and to anticipate everything correctly. Assumptions are made, surprises - things not thought of - arise, and there are unintended consequences. It is not always easy or possible to fully grasp the consumers' perceptions of a product, or its potential. Over time, as things transpire, the eventual solution may be significantly different from the initial solution. In many cases, the original solution is merely a spring board that get the innovator in the arena. Engagement with the problem and its solution often gives new direction and may open up more or new opportunities. Arriving at the eventual solution is an iterative process - a lot of learning, original thought and decision-making take place.

The innovator learns a lot as he moves along. He comes across easier, cheaper and better ways to solve the problem, and practicalities and constraints become evident. Still, it is advised to get market feedback as soon as possible. The innovator needs to get the balance right between reasoning or analyzing too much and too little. He should work with and from feedback on a minimum viable product (MVP), rather than making too many design changes or improvements before taking it to the market first. He should maintain balance and must not over-perform on the solution: he should know when a solution is good enough, and not try to add all the bells and whistles initially, because it might be nice, but redundant. Subsequently, he can further work on optimizing the solution. The innovator critiques and questions the solution to move it forward and to determine whether it can be improved still. The end goal is a solution with fair price and (for) quality - also in terms of what the customer sees as adequate quality.

\subsection{Method and Technique}

In many cases, the innovator would perceive the problem and quickly comprehend a general solution. He will then work on the details of realizing it. The innovator works a lot from improvisation and assumptions. In some cases, it is important to keep some momentum, and when necessary, the innovator will keep jumping between different perspectives. A lot of learning takes place while the innovator works on solutions. Learning forms a crucial part of arriving at solutions. The right conditions and environment must exist for learning to take place. Learning builds on previous learning and expands this way. Learning allows further and additional learning.

Of course, a strong link exists between creativity and problem solving - creativity and problem solving have a lot in common, and problem solving significantly helps to explain creativity. It is seen as important to make a decision to start - to take action and to start somewhere. Whether the start is wrong is not really important. This simply reflects on the fact that creativity involves a lot of learning and original thought. Indeed, a lot of learning occurs during creativity. The innovator is essentially learning a technology, etc., or upskilling himself in terms of a technology, etc. Proper leadership is also crucial: It is important to know when to lead and when to follow - when to turn to others to guide you.

An overall objective is to get both the technical aspect and people, social or business aspect of innovation right. The innovator must be mindful of competitors and existing solutions or substitutes, must know the specific market segment he is targeting, and must ensure that his solution is not over- or underdesigned. 


\subsubsection{Information Collection and Research}

As subsequently discussed, a key constituent of creativity is perspective formation. To this end, the innovator collects information in a number of ways. Popular and common sources of knowledge are: 1) reading; 2) conducting research: this may entail background research or more extensive research, and particularly done via the internet; 3) people: friends, peers, experts, networks; 4) customers.

The objective may simply be to fill in a blank, or, something more substantial, like sourcing an idea that can instigate a solution. When starting work on a problem, the unknowns and assumptions are typically defined early on, to make work of resolving these.

Talking to a lot of people and getting the views and opinions of a lot of other people are seen as important, as the innovator is limited in background and thus what he knows. Talking to others helps to expand his perspective.

Some build a resource or support system around them, comprising of information and people. This too can accelerate the efforts of the innovator. The innovator may equally draw from knowledge and experience he has built up over time. In this sense, (having built) a general or broad knowledge base is seen as beneficial. It may be through seemingly an unconventional source or medium as watching documentaries.

Research is seen as a good tool to understand and make sense of the problem and to get under the hood of a new idea. A simple search on the internet can provide substantial background information. Research can also quickly establish the uniqueness of an idea, which is crucial. It must be ensured that the idea is indeed an inventive step - it should not be obvious to a person skilled in the art. To go deeper, more specific research is conducted.

Conducting surveys and getting feedback from early adopters are examples of collecting information from customers. Customers are also typically approached with minimum viable products, to obtain feedback.

The innovator can quickly determine how much external help he requires, particularly in the form of experts. He may associate with people knowledgeable - expert - in the discipline. He may consult with his network or peers - both internal or external to his organization - to help point him in the right direction.

Simply a proper culture - an informal work structure or arrangement - that encourages communication and sharing of ideas, may significantly contribute to sourcing the information required.

Conferences are also seen as a good source of information, particularly in terms of gauging an industry. The subsequent objective may even be to also speak at conferences as soon as possible, to further information gathering.

The innovator may find the thoughts of others who have just started with something innovative, and who have filed a patent, inspiring, particularly when searching for ideas, rather than specific answers to solution details.

\subsubsection{Forming Perspective and Sense Making}

Innovators use a number of means to build perspective, and they frequently shift between the means. It may vary and be diverse as drawing doodles, making word lists, and doing some research. The general objective is to think of something else, or something different, or to break with/ from the predominant view, perspective or mindset, in order to advance it. Perspective is a lot about sense-making and also internalizing the problem. During perspective formation, some employ whole brain thinking - engaging the left brain and the right brain simultaneously. The innovator generally has an idea of the end goal or outcome before he starts. When innovators get stuck with a problem or its solution, and struggle to move it forward, they would increasingly shift gears - use different tools, etc - to shift perspective.

The innovator may use metaphors and analogies for perspective and inspiration. Telling stories are an example of this. He may ask (certain) questions, to force the brain to think of something else/ different. He may look at other sources to break perspective. A number of participants report an extensive array of creative thinking tools that they use. A toolbox of multiple tools is useful, given that each tool has a particular outcome. A tool that is great in one context may be poor in another. They will search for the right, applicable tool, or homebrew tool - composite tool - that will advance perspective. The tool may as much depend on the particular innovator, as the problem. Tools are an easy way to start. They highlight some of the principles at play, and help with quick perspective formation. Tools that aid perspective may be as diverse as (measured) trends that have proved to be stable over time, and a number of external or abstract sources may provide perspective through analogies. Some prefer to come up with a diagram or representation of the problem. They may also build a model or prototype to look at aspects of the problem, also from a practical perspective, in addition to a mental perspective.

Some use triggering, with specific triggers designed to change the way of thinking away from the norm. Random words or random images are examples of triggers. Reframing the question or problem, or 
putting the same problem into a completely different context may also help to break a predominant or dominant mindset. Putting the problem into a similar context that have been solved before helps with context and to define the problem. Innovators may equally be cognizant of their or others' perspectives, so that they can find a way to bring them out of it, in order to challenge it. They look for ways to break familiarity with, preconceptions of, and even prejudice towards a problem. This may entail asking stupid questions, and being ridiculous and unrealistic, in order to get rid of mental filters, and to return to a sense of cognitive innocence or neutrality. Generalization of the language - for example, speaking of better visuals, rather than a better display - is another way to advance perspective, given that language restricts the solution space. (Simultaneously) working with multiple solutions is another way of looking at the problem from many different perspectives.

Although perhaps more analytical in nature, any way to deliberate the problem may help to unpack the problem, make sense of the problem, and order thoughts regarding the problem, and thus aid perspective. Examples are: a think-through, reflection (reflecting on the problem), writing, debating the problem with people, and conversing the problem with others.

The innovator may be able to intuitively provide a different perspective - he may draw on his intuition to give or shift perspective. In these cases, it is mostly a case of realizing or materializing the intuitive thought. Writing, drawing, thinking on paper, and putting down on paper what is in the head may be examples of this.

Innovators may also shift or iterate between the left and right brain as a way to advance perspective. For example, they may draw the problem, to force their brain to go into the creative thinking mode. Then they enter into an analytical thinking mode, to criticize the drawing. They then again redraw. This way they can progress their thinking along quite a focused projectile. Another way to shift perspective is to unpack a problem, and to then follow a process of repackaging it, as a way to see how it is understood, and what differences surface. Edward de Bono's thinking hats is another example of shifting perspective, by changing the type of reasoning or thinking applied to the problem at a particular point in time.

They frequently shift perspective in the sense of zooming in and out of the problem: they start with an overall view or big picture, before they zoom into details, rather than the converse. They are able to grasp the big picture rather quickly. They prefer to know a little about a lot, rather than a lot about a few things. They keep on refining their depiction or representation of the problem, by zooming in - expanding and narrowing down - more and more. Once they have a good idea or sense of the problem and solution, the will start with more technical discussions. They break down huge objectives into much smaller deliverables, and draw on them around them to creatively solve the deliverables. They may simply again distance themselves from zoom out of - a problem, if they are unable to come up with a solution for it. The objective may simply be not to see things from the ground too much. This form of abstraction also helps to form perspective.

The knowledge and experience they have built up, can greatly help them with forming perspective. They internalize the models and tools. They learn by facing new challenges, and may learn through helping and training others. It equally challenges them, and helps them to point out gaps in their knowledge. An inquisitive mind, for example, wanting to know why things are not working like they are supposed to, also help them to build up their knowledge.

During the process a lot of synthesis also takes place, and this may occur simultaneously. For example, (bits of) imagination, experience, and reasoning may all be synthesized into a coherent whole.

Working on a problem for an extensive amount of time will also cause knowledge of the problem to increase. The innovator becomes more knowledgeable on the problem and its potential solutions, and soon finds easier and better solutions.

\subsubsection{Prototyping}

Prototyping - a minimum viable product (MVP) - allows the innovator to quickly get the essence right. Prototyping helps to surface assumptions and unknowns, and provides a model, representation, and also a visualization, of the solution and its environment. It helps to articulate the solution, and it provides an interaction and experience with the solution, and it can be used to engage (the input of) key stakeholders, like potential customers. This can help to improve the alignment, accuracy and relevance of the solution, and its completion time. It also provides a model to evaluate against, and permits testing the implementation, and noting how the solution practically works. Getting feedback, particularly from potential customers, as soon as possible is seen as critical. The objective is to balance reasoning, thinking, designing and planning with prototyping to equally introduce the practical aspects. Plausibility of ideas, designs, etc. can be checked by speaking to experts and users.

Prototyping is also useful when the innovator wishes to further understand or develop the problem or solution. He more or less knows the outcome that he wants, and has an idea of how to accomplish or realize it, 
but is not completely certain. He will simply try and see, it is a way to get him going, and it may provide him with something to run with. Instead of analyzing the problem, he can engage in and work on something practical - a practical solution right away. Failure is seen as constructive, and providing direction. As simple a prototype as possible is built. Learning occurs through practice.

Some of the criteria considered as part of minimum feasibility are: 1) effort and revenue; 2) attractiveness and potential; 3) uncertainty versus risk, 4) income timelines, and 5) long term profitability.

\subsection{Immersion}

In addition to thinking about the problem or solution, some innovators like to involve their senses: observing, looking, and listening. Instead of just sitting and thinking about the matter, they like to do something - to touch and feel things or something. Or, to take a pen and to simply write an idea on a piece of paper, as a way of expression themselves. To innovators who are tactile, senses are very important. Some argue that you are most creative when all of your senses are completely awake/ involved. It is about fully present in the moment or the continuum of moment, and taking in everything that is going on.

A principal argument is that you can have the full experience or perception through learning (reading) only. Thus, creativity is as much about the senses. Phenomena must also be perceived through the senses to be really understood. Coffee and tea, and Japanese tea ceremonies are classical examples. It will never be possible to fully understand coffee and coffee drinking through books, etc. alone. The problem is experienced first hand. Something like Japanese tea ceremonies may incorporate additional elements - like spiritual elements - over and above already involving all 5 senses. Involving the senses can invoke emotions with the innovator, that can further contribute to creativity. The problem or solution can be better understood, based on the feelings it invokes - how it makes the person feel. Related to this, people's experiences of problems are equally important. Interacting with the problem, or its solution, is seen as important - it allows the innovator to sense and perceive the solution, and he essentially gathers information this way.

Play is also seen as a very prominent stimulant of creativity and creative thought. Play allows free reigns, and allows the person to be a kid again: there are not stereotypes, preconceptions, or boundaries. It can also be seen as a way or method to take the person out of his natural environment - out of what is known or familiar to him, and his routines and thought patterns. Play can lead to discovery. Play may be a form of informal prototyping.

Sometimes, it may be necessary to tune down the analytical part of the brain to come up with solutions. In addition to this, what further distinguishes the innovator is switching between sensing or experiencing and thinking - knowing when to involve and suppress the analytical (senses).

Immersion has an element of intuition to it. The innovator may interact with a problem by battling with it on paper. Interaction with the problem may also entail (visual) representation, including doodling and drawing. To this end, things like A3 paper, colours and sticky notes are commonly used. Some view writing or typing as too detached and thus inferior. The former (paper, etc.) is seen as tactile and physical, and the latter (typing, etc.) as cold and hard and clinical. The process normally involves a lot of chaos and rework.

\subsection{Imagination}

The role of the imagination during creativity is seen to be mostly visualize, model and simulate solutions and ideas. Visualizing the solution helps to demarcate its functionality, properties, attributes, and aesthetics, particularly its physical properties. It helps to articulate the solution or problem. The ability to visualize an idea or solution helps to determine whether it is practical and feasible. The innovator can typically see - visualize - the complete product before it is built. That vision is then commonly broken down into smaller, manageable parts and deliverables. Visualizing the solution helps to (be able to) create it. It provides a basis to work from, and helps to define what to work towards, and how to work towards it. Visualizing the solution may also help to further develop and advance it, and the innovator may combine visualizing the solution with other tools and techniques, etc.

Imagination as simulations and visualizations of scenarios and events, may also be used to better understand problems, helping the innovator to consider things like cause and effect, and social contexts. Metaphors and analogical reasoning can be used to represent abstract concepts, to better conceptualize and articulate them, and to see how one thing relates and applies to another. There are triggers and tools to stimulate or advance the imagination, and the innovator may be visually literate and trained.

\subsection{Intuition and the Sub-Conscious}

\subsubsection{The Role or Contribution of the Intuition}

Intuition is largely seen to be based on experience. It comes from making mistakes, learning from it, 
and developing experience from it, to later re-apply when solving problems. The sub-conscious works to answer conscious and sub-conscious questions regarding a problem. The sub-conscious is rather persistent, and reliable in this sense. The brain is taken to be very good at making connections between things that are completely unrelated or removed, and this occurs in the sub-conscious. The sub-conscious of course does not require focus to function. Ideas and thoughts germinate in the sub-conscious, and it is difficult to know how long an idea has been germinating. Many times, questions point to knowledge or comprehension gaps that must be filled or closed. When a problem is known or partly known, it is not too difficult for the brain to come up with its solution. By being curious or interested in a problem and its solution, the innovator is essentially writing a letter to his mind - requesting his mind (sub-conscious) to work on the problem and its solution. This way, he is actively involving the sub-conscious, or switching to the sub-conscious. The brain attempts to subconsciously solve problems, and the innovator allows his mind room to sub-consciously work on the problem.

Intuition may play a key role in advancing problems in their initial stage. Often times solutions to problems have an intuitive crumb-trial. A number of disciplines and input are mixed and synthesized, a story, model or representation is advanced, and initially the process may advance more questions than answers. The innovator may have a vague idea or solution, or a vague problem statement. He is aware of it, but do not know the imminent answer. He may only know the general solution or direction at that time. And clarity only comes with time. It generally points to a lot of questions, uncertainty and direction that must be resolved during the process. Even if the innovator does not know the solution, he only needs to know how to advance and work towards a solution. In this sense, the intuition and sub-conscious, and in turn perspective switching, play an important role. Perspective switching, the intuition and the sub-conscious may form a synergy: perspective switching and the intuition may provide the sub-conscious with enough to in turn provide additional content to work with.

The innovator develops and makes use of senses - a sense of the problem, the context, the perceptions of people, like customers. He may develop a sense of what may or what may not work. The innovator may use his intuition to evaluate and assess something, to form an opinion of it, and to decide on the direction to take - where to look at and what attention should be paid to.

Their intuition may offer them a glimpse, sense or feeling of an idea or possible solution to a problem, and perhaps the best way to move forward, as well as what to avoid. They may also focus on aspects of an idea or solution that essentially bother them and may pay attention to it and keep on exploring, until they have no more questions about the problem or solution, or until they can shake off the sense of discomfort, unease, or concern.

The sub-conscious may equally realize or perceive problems before the conscious does or are aware of them. The innovator may later on realize that he was or is grappling with a particular problem, or better understand the dimensions of a problem. In general, he may register ideas, and only then realize that it links to a problem that he had not even articulated (properly) yet.

\subsubsection{Engaging the Intuition}

Most innovators do not find it difficult to draw from their intuition and sub-conscious, and to let their intuition and sub-conscious contribute to the solution. They learn the art of quieting themselves, and they are willing to listen to their intuition. They simply switch off and free their mind, walk away from difficult problems for a while, and focus on something else for a time. They will also use techniques like self-distraction - deliberately distracting their focus, not overly paying too much attention to something or some aspect for too long, and consciously engage themselves in multiple activities or thoughts, or jump between perspectives or objects under consideration. They typically play with things - for example, flicking a pen - whilst thinking on something or discussing something. They mull over problems, ideas and solutions, but also allow time for ideas or solutions to gestate. They typically find that answers and solutions come to them when their mind is generally pre-occupied with something else - being in the shower, in traffic, or walking on the beach. It may be that the more enjoyable the pre-occupation - time in the shower as opposed to time in traffic - the more active the intuition and sub-conscious become.

There are a number of ways to further stimulate the intuition and sub-conscious, and innovators knowingly or unknowingly do so. They will charge their sub-conscious, by going through a problem well ahead of time, or before bedtime, and some time later solutions and ideas will come to them. They frequently find that inputs and events from the environment stimulate and trigger their sub-conscious and intuition. They will notice, witness or read something, in a number of cases unexpectedly or unplanned. They may actively stimulate their sub-conscious by switching their environment (going for a walk, visiting a completely new environment, etc.), or exposing themselves to new input (reading a book, paging through a magazine, chatting with people, etc.). Random stimulants are able to trigger solutions, because they trigger a train of thought and 
serve as a critical input to the sub-conscious. Any input that can trigger a completely alternative vantage point or perspective, may be relevant. Some things, like music and art, seemingly help to stimulate the sub-conscious.

Innovators have a number of outlets to give expression to their intuition. Post-it notes and drawings would be examples, and the process is generally very artistic and fluent. They give free reign to thoughts, expand on thoughts and see where it goes.

Innovators may rely on their intuition to come up with solutions and ideas and may indeed source ideas and solutions through their intuition, but ultimately commit to a much more methodological and structured approach to working on ideas and solutions. Ideas and solutions emanating from the intuition are still subjected to objective and systematic evaluation. The innovator still backs his intuition up with intelligence and data. His intuition may not always be right, and he may overly rely on his intuition.

\subsubsection{Attributes of Intuition}

The innovator may experience periods or intervals of a creative streak or creative rush. He may also be more creative when he is or needs to pass time, or when he is relaxed. He also gets a lot of ideas when he is actively working on a problem - this may be related to a fresh problem, or a fresh mind. Ideas may come to him out of the blue, and innovators love to journal their ideas. The more the innovator understands the problem, the more he can do with it, and the more his intuition can contribute. There are a number of triggers that shape ideas, like people or events, and his ideas and thinking generally evolves, also being shaped by feedback.

An innovator may not obtain a solution, because the time may simply not be right, many times implying that something is missing - like a key perspective. He may even know what may be lacking, but not know how to resolve it. Innovators love to keep a list or record of projects that are not ready yet, and that are waiting for something to dawn.

The innovator may over-engage his mind on a problem, and this is generally what most of them are aware of, and try to avoid. A classical example mentioned by one participant is over-playing a musical piece. The performance with which a musical piece was played, deteriorated after it was mastered. Leaving the musical piece for a while restores the performance. It restores originality, freshness, and newness of the problem and solution. It may also be a case of having exhausted perspective, and requiring a new perspective or input to be able to be innovative and creative about the problem or solution again. The innovator typically seeks to balance his work or tasks with contrast.

Rather than forcing his brain to go down the same path over and over, and thinking analytically, the inventor will relax and silence his mind, in order to switch to more intuitive thinking, to discover the next step or a possible next step. The innovator may use different activities - diverging his attention to other or different activities - not necessarily related to the problem at hand, as a way to switch from more analytical or conscious thinking, to more intuitive or sub-conscious thinking, and to move the problem from the front of his mind, to the back of his mind. Sometimes it helps to be all over something - the problem or solution. It also serves as a way to step away from a problem and revisit it.

Great uncertainty and initially unsolvable problems are antecedents of aha moments - it is only these and such problems that can really cause aha moments; minor problems do not really cause aha moments. It relates to the extent of the gap between the problem and the solution - the amount of effort that was required and the level of immersion in the problem. Aha moments are thus relatively expensive. Aha moments are also the consequence of deliberate thinking - sub-conscious mental activity - on a solution to the problem at hand. The fact that aha moments come at random times, and that it is not always possible to explain the steps to a solution, demonstrates the involvement of the sub-conscious.

Intuition may not always be regarded as practical, and thus valued. It may be seen as useless, particularly when the link to creativity is not made. The innovator may also practice and learn to utilize his intuition.

\subsection{Problem Statement}

Views are split as to what extent it is necessary to define the problem beforehand. Some wish to exactly understand and define the problem. It gives them something to focus on, work towards, and attempt to solve. It allows the innovator to begin with the end in mind, and it keeps him from solving the wrong problem. The problem or idea must first be understood, before it can be solved. If the problem is not understood, it can not be articulated well, and it is not possible to start solving it. Problems define, call for, and initiate the need to be creative, and render creativity practical. Problem statements help to prioritize work and to determine which projects are relevant, and to select projects to work on. They help to understand the extent of the problem, and to plan for it. Defining the problem may help to source possible (existing) solutions for it. Problems exist because an objective or goal is realized, with an obstacle in the way of reaching it. Without a problem statement 
or definition, there is little direction, and a lot of distraction - it becomes easy to be side-tracked and to chase the wrong objectives. If the desire is to actually solve the problem, it is very important to define the problem. On the other hand, if the innovator just want to play around with the problem, and possible solutions to it, it is less important. The solution is often times within the problem. The more the problem is explored, the more likely a solution is going to be found.

Others see it as only necessary or important to loosely or informally define the problem. Defining the problem is akin to defining creativity, and creativity can not be defined, but only unleashed. It may be necessary to articulate the problem, but it may not be necessary to do so explicitly, precisely or narrowly. A loose or fluid problem statement may very well suffice. It may not always be possible to fully grasp what the problem actually is, or only a feeling or sense of what the problem is may exist, particularly during initial stages, and a problem statement may then be hindering. Room is allowed for experimentation, and for the problem and solution to develop and take direction, and the process also has to encourage it. The view is that, the moment a problem statement or problem definition is given or noted, the outcome or direction is decided, hence there is little left to explore. It merely becomes a case of finding a solution for the stated problem. The problem statement or definition can be left for the end. In essence, the solution may very well influence and impact the problem and problem definition. Defining the problem too much essentially constraints the solution space. Defining the problem limits the solutions or confines the solution and thinking about the solution. It is possible to think too rational about the problem and its solution, and it may be necessary to rethink or re-conceptualize the problem - to make it about something else.

Generally, the overall objective is to understand the problem as much and in as much depth as possible. There may be no formal process to defining the problem. The view is also that there are different layers or dimensions to the problem. The general problem may be understood, but not the specific problem. The problem also involves aspects like the business aspect, and the unique value proposition. It may require determination to fully grasp the problem. Aircraft accident investigations are cited as the ideal example, in that they consistently seem to get to the root.

\subsection{Building on Past Solutions}

Again, two opposing views surface. Some feel that preceding work and past solutions are useful, others feel they are restrictive.

Those that break from past solutions wish to see each problem as unique. Reverting to past solutions can render the innovator semi-blind and can obstruct creativity. If he looks to past solutions, the innovator is not seeing the problem for what it is.

Those that utilize and draw from past solutions, get inspiration, input, perspective and direction from previous work. It is seen as important to involve experience as well. It is argued that one can still start on a blank page - fresh - when incorporating previous knowledge and experience. Incorporating previous knowledge and experience gives the innovator and advantage, and may save time and effort. There may be similarities in problems or solutions. Any solution builds on building blocks. Reusing building blocks have significant benefits: it prevents reinventing the wheel, helps efficiency, and may even lead to better solutions. The innovator can build on lessons from the past. Past knowledge and experience become part of the innovator's tacit knowledge and experience, and he becomes smarter and more experienced by the day.

The subsequent argument then is that it would be impossible to separate the innovator from his past knowledge and experience. By implication, this has positive and negative consequences. Past experience greatly shapes perspective and decision-making. Past experience and knowledge can both positively and negatively contribute to creativity and innovation - it may both quicken creativity and innovation, or stifle it. In light of the above, it is clear that neither position - breaking from or building on past solutions - is conclusive. The problem at hand may determine the best approach. There may be technical reasons for building on known solutions, and there may be clear reasons for throwing everything out.

In a way, there may be differences between past solutions, and past catalysts of solutions - creative moments of the past, and how it was realized. This may help the innovator to be creative again, and to again engage, release or emit creativity. The innovator does not have to be original in method, to be still original in the solution.

\subsection{The Solution Space and the Number of Solutions}

The overall view is that there are multiple - or even infinite - solutions, and the innovator generally must choose the best one. The shotgun approach is also frequently mentioned - innovators, typically novice ones, will try several directions in hope of finding a solution, and will then run with the first workable solution. The solution generally must meet and be optimized along certain criteria and requirements. Additional 
evaluation criteria may also emerge and become apparent during the creative and innovation process. The process of finding the best solution frequently involves trade-offs and is commonly not an easy task. Innovators typically stop at the first solution, without considering and evaluating alternative solutions, to choose the best solution among several candidate solutions. Some success can be the enemy of a good success. Good success mostly comes from a more deliberate effort. Enjoying some success may rob the innovator of greater success.

Factors like time, money, and experience of solutions may also influence the solution selected in the end. Solutions are typically evaluated against cost, flexibility, quality and value addition. Considering alternative solutions and several solutions increases the efficiency of the solution. Subsequent solutions may improve on initial solutions. With subsequent solutions it is commonly found that there are multiple solutions to the problem, and better ways of solving the problem. Thinking in terms of having arrived at a solution closes off thinking on further improvement. The innovator should accept that there are likely multiple solutions, and that it will be necessary to find the best solution.

Techniques like expansion-consolidation helps to fail solutions quickly, in order to move the solution forward more speedily, and to remove issues as soon as possible. The solution is expanded, evaluated, and consolidated, and this process is repeated. It also helps to surface the fundamental dynamics the problem pivot on.

A solution also does not have to be the best solution, but the first solution that the market will accept. Thus, another approach is to define the ideal solution, and to work backwards to the first solution that the market will accept.

\subsection{Growth and Development}

Interestingly, the respondents did not readily change their learning style during the creative and innovative process. They stuck to the method and process they knew to approach, interrogate, unpack, and work problems. The innovator learns from his thinking and work and builds up experience and expertise. At the same time, only when they reflect, do innovators really learn from the creative process. During the creative process, they are mostly applying what they have already learnt.

\subsection{Teams}

Whether the innovator prefer to work in a team, or on his own, depends much on his personality, and the stage of his idea or solution. Some innovators can be very creative either by themselves, or in groups. Some innovators see their creative space as very personal. There are aspects of creative work that the innovator best should do on his own, and there are aspects where he should best involve others. Composing music is an example of creative work that cannot really be done in a group.

The innovator must be open in the sense of believing that he can and will benefit from the input and perspective of others. New insight can come for diverse sources, like 3-year olds asking relevant questions. The other party or parties will look at the problem or solution in a different way, or ask a different or new question, and this may trigger something with the innovator, so that he too can look at or think about it in a different or new way. Feedback and collaboration should have an outcome and result that is bigger than the contribution of any individual in the case of groups, or that of the innovator or the other party or parties, in the case of the individual working on his own. Sometimes the innovator himself may not (initially) look at the problem or solution in the best way or ask the right questions. The innovator generally wants someone that will look at the problem or solution differently, so that he and his thinking is challenged by this, and so that his thinking can develop from this. For the same reasons, the innovator prefers and seeks a group where he will be challenged, asked questions, and his suggestions are challenged.

Teams help to contribute a diversity of perspectives on the problem and solution. Groups allow sharing of ideas and collaboration. It may also permit out of the box thinking. Groups are able to overcome established ways of thinking - paradigmatic thinking - because they are not subject to the same culture of thinking, and typically manage to break it. Groups may achieve and emit high levels of creative energy. To do so, there must be a lot of freedom in the group. It must be possible to entertain absurd ideas in the group. Entertaining absurd ideas or questions that are naturally not possible (by current standards) shapes the train of thought or thinking. The setup of the team influences its overall perspective, and this influences the outcome. Teams comprising of individuals that generally share a common perspective, allows more specialized and smarter or more clever thinking. Teams comprising of individuals with different or divergent perspectives, allows more divergent thinking.

A general condition of teams is the ability to work with others. It is possible to be constrained by the team and a team. It must be possible to freely channel opinions, and to develop opinions into something more. Team members must be allowed enough room when there is still relatively little in terms of a solution, and 
initial participation and contribution must be steered. It is important that group participation is authentic. With a team, it is necessary to get everyone on board first, and to establish a collective focus - to get everyone to focus. The process must be properly structured, managed and directed, also so that it properly unfolds and develops. The wrong input at a time may ruin the process, for example, critiquing when it is still time to think creatively. The group and process must be facilitated, by discerning the direction the group is taking, and steering it. It is necessary to both allow the group freedom, and to reign it in, at times. Some tools are not well suited for groups, because they are too unstructured. The wrong participants in a group - for example, people who are negative or emotional - can also compromise the outcome. Not everyone in the group may be on the same page. Political and personal agendas may also surface in groups. Certain personalities may find it difficult to work together. With groups, it may also be necessary to upset the status quo, to truly get an unique or different outcome. The same people may continually participate in the group, leading to the same outcomes. Normal, conventional, or routine thinking must be broken up.

Working on their own, innovators are generally able to work much faster, and enjoy greater freedom to change direction and consider alternatives. They are less rushed and under pressure. They may not always enjoy having people around them. Still, listening to others' perspectives can help the innovator's own perspective. Innovators tend to prefer to do a lot of work beforehand, before articulating and presenting an idea to others. When the time is right, particularly when the time is right for critique, the innovator will approach and open up to others. The extent to which others can add to the idea or solution equally depends on how far the innovator has already advanced it. Others may not understand the problem or solution fully, because it is too early. The innovator must be able to comprehensively understand and articulate it himself first. The innovator is rather vulnerable while he is creating, and involving others too early can hurt or destroy the creative attempt - prevent the innovator from giving it a try, when giving it a try and working on it is the best option and advice at the time. At the same time, involving critique early on in the process, may spare the innovator a lot of wasted work later on in the process.

The wrong feedback can discourage, distract and confuse the innovator, and he must be able to manage and assess the input. Input is not straight forward. The innovator must find the right person to bounce ideas off, and to constructively critique the solution. The innovator must be able to know how to select people to approach - what the person could possibly contribute. Different personalities may take different vantage points, focus on different points, and give different assessments and critique. Other parties are not always relevant to innovation and the creative process, and others must be on the same wavelength to really contribute to the topic or suggestion, and to be inspiring. The innovator may find it draining to work with people who are not in the same frame of mind. Their background, training, experience, commitment, and interest play a big role. The emotional state and vantage point of the other party or parties can also have a significant impact. Sometimes the other party may not understand the problem or solution at all, or they may not share the vision of the innovator. Sometimes the problem or solution must be experienced, rather than explained, to be fully appreciated, and the other party may lack the appropriate knowledge to be a true critic. They may simply not be able to keep up with the innovator.

An important issue, both in teams and the innovator as individual approaching others, is the level of freedom the innovator is allowed or can expect - whether the innovator is able to let his heart and mind go, and how things, like emotions, would be handled and approached. The innovator must feel that he can let his mind and heart go, because there is no cost or objective associated with it.

Some may not be able to collaborate at all, whilst others will be perfectly happy to informally and openly chat about something. Feedback and input from others may be as simple as an informal conversation. Having people think differently and work differently, can be immensely frustrating, but also stimulating, and more creative. And sometimes working with people who are aligned and thinking in a similar way, can be more productive. The innovator may need to select the experience that will benefit him most. Incorporating others may also help to secure buy-in and commitment. Given the skills required by the problem or solution, the innovator may be forced to work with others. The innovator may benefit from establish good relationships with people that can provide him with input and perspective.

\subsection{Succeeding in the Market}

A primary attribute is whether the idea or innovation has business sense or a value proposition. Whether the innovation is functional is secondary to this. A fine balance between the business side and creativity is required. The innovation must be practical, relevant, and the market must want to buy the innovation. Things like brand or image, or creating a desire for the innovation, may be as important or more important than functionality or being the best functionally.

Innovation also requires good understanding of the market, and how the market functions. The 
innovator should stay ahead of times. The innovator must (be able to) disrupt and overcome existing structures and politics in the market. It may be difficult to supplant an existing, strong brand in the market. The innovator will face resistance from incumbents. The innovator must realize that he is not alone in the game. He should know his competitors, and their own innovation plans. Critical thinking is seen as an important skill for survival. It is a matter of not just coming up with a solution, but exceptional solutions - extensively and fundamentally rethinking solutions. An existing solution or product may be made irrelevant, by redefining it by asking different questions and creating different perceptions or occupying different spaces. It may be necessary to go beyond obvious answers or solutions. This requires a willingness to spend enough time and effort and being willing to work through the obstacles of a potential answer or solution, to properly interrogate it. A solution that is difficult, hard, and frustrating is seen as a good sign.

It can be difficult to bring something to market that is truly unique or radical. It may be difficult to know what customers want and focusing on the customer is a far more credible way of innovating. The market has its own opinion of what is innovative, and what is not. Also, the market's opinion of what is innovative is also shaped, and not necessarily left to them to decide themselves. Thus, it becomes important to know what to pitch to whom and how. The innovator must be able to connect with his audience and capture and hold interest.

Innovation is also fast-paced, prompt or punctual. It is very in the moment, what or who is present or relevant in the moment, and what the innovator can combine and come up with at the very moment. It is important to get the sequence and timing right.

At the same time, markets may be short-sighted - markets currently may be driven by quick fixes and pushing current business to a large extent, and not future business per se, and this may be limiting innovation. The innovator may benefit from looking to reverse this approach and culture, and by working on actual, current problems with solutions that is more than just quick fixes.

The innovator's emotional state, psyche and environment significantly affects his outlook and ability, and he requires emotional intelligence. The innovator should manage his network - he must surround himself with the right people. The innovator must have a vision, be determined and set on achieving it, and must actually refuse to accept that it cannot be attained. The innovator should guard against being overwhelmed by the problem and sourcing a solution for it. He should remain objective about a problem and should avoid carrying prejudice regarding the problem or its solution. The innovator should not shy away from big problems. Daunting problems still require practical solutions.

The innovator's experience counts in his favour. He must be able to evaluate and assess ideas and opportunities, their feasibility and uniqueness (unique value proposition), the inherent uncertainty that must still be resolved, and the knowledge and information available to the innovator. It is important for the innovator to accumulate practice and experience.

Innovation requires proper planning and effort. It is important for the innovator to be realistic, and to engage in reality checks. Not only due to the effort, time, money and risk involved, but also not to miss the market. The innovator must consider scaling his innovation effort. It may help to first start with something small and attempt to get that right. Incremental innovation is perhaps easier to get right than radical innovation. Innovation may seem easy, because it may seem that solutions come in a blink, and when disregarding the time and effort it took to prepare the ground for the solution. Innovation requires commitment and dedication over an extensive period of time. This may have certain demands, consequences and implications, for example, financial implications. Innovation requires dedication. The innovator should be consistent in his effort and see it through - he should not jump around between solutions. A lack of resources may cause the innovator to grab and run with the first solution, because he cannot properly invest in solutions.

Implementation and being able to implement is just as important as being able to come up with new solutions. Implementation may be demanding. The innovator may benefit from partnerships to help with execution and implementation.

\section{Conclusion}

Several attributes of creativity were delineated. Creativity is a lot about different and new thinking. Perspective plays a key role. It requires a lot of freedom. True creativity merges the left and right brain. Some of the key attributes are: creativity is 1) contextual, 2) cultivated, 3) habitual, 4) proactive, 5) purposeful, 6) directional, 7) intuitive, 8) confident and assured, 9) aware, 10) non-logical,11) expensive, and 12) grounded.

It is possible to pick up when a person is creative, and to discern creative people from non-creative people. A number of factors demonstrate a person's creativity: 1) how intuitive they are, 2) how much foresight they have, 3) how proactive they are, 4) how much initiative they show, 5) how open a person is to suggestion, 
6) how willing a person is to explore, 7) their language and (level of) thinking, 8) mental predisposition, actions and behaviour, 9) the ability to deal and cope with ambiguity, and 9) originality and thoroughness of thought. Simple tests can also reveal creativity.

Some may be naturally creative. Still, creativity can be developed through practice, and education or upbringing plays a significant role when it comes to creativity. Much of creativity entails becoming a better thinker. At a certain point, meta-creativity - understanding of creativity - rather than creativity may develop further. Creative expertise also develops - the innovator increases his array of tools, and transition between them more fluently and effortlessly.

Common stimulants of creativity include simply being truly inquisitive, aligning the mind and emotions, and stimulating the sub-conscious and intuition. Alignment of the mind involves clearing it, quieting it down and relaxing it, and opening it up. The sub-conscious and intuition can be stimulated through collection general knowledge, visualization and imagination, a change in environment and any related or similar trigger - any deliberate or unplanned real life experience serving as a trigger. Creativity breeds creativity - it helps when the person enjoys being creative. Problems or problem solving - being faced with or wanting to solve problems - also aids creativity. Emotions and circumstances like passion, necessity, stress, pressure, fear and desperation can also contribute to creativity. The innovator or entrepreneur may quickly recover when faced with adversity and start to see and approach it as a challenge. At the same time, creativity is also seen as strategic or planned. Creativity or creative thinking can be (made) systematic, with routine to it, and tools to aid creative thinking. The innovator or entrepreneur may deliberately sit down to be creative, and to do creative work, using creativity tools and methods. The environment, (interaction with the right) people, mindset, cognitive development, and aspiration (motivation) have a significant impact on creativity.

Creativity allows a semi-formal process. Although there is no cut-and-dry process, the innovator has a general routine, procedure or outline he normally follows during the creative process, that is rather standard. There are several distinct modules or processes that his thinking progresses through or along. Some of the steps involve contextualization, information and knowledge gathering, perspective formation, and conceptualization. Implementation of a creative idea or solution forms a significant part of the creative process, and it involves very little creative thought.

The innovator's perception of the problem will likely develop and evolve as he works on the problem. Consequently, the eventual solution may end up significantly different than the initial perception thereof. In many cases, the original solution is merely a spring board that get the innovator in the arena - to get him going. Arriving at the eventual solution is an iterative process - a lot of learning, original thought and decision-making take place.

In many cases, the innovator would perceive the problem and quickly comprehend a general solution. He will then work on the details of realizing it. The innovator works a lot from improvisation and assumptions. In some cases, the innovator will keep jumping between different perspectives, as a way to maintain momentum in working on a solution. A lot of learning takes place while the innovator works on solutions. Learning forms a crucial part of arriving at solutions. Creativity and problem solving have a lot in common. It is seen as important to decide to start - to take action and to start somewhere.

A key constituent of creativity is perspective formation. There are several ways through which the innovator can broaden his perspective. Innovators use a number of means to build perspective, and they frequently shift between the means. The general objective is to think of something else, or something different, or to break with/ from the predominant view, perspective or mindset, in order to advance it. Perspective is a lot about sense-making and also internalizing the problem. During perspective formation, some employ whole brain thinking - engaging the left brain and the right brain simultaneously. The innovator generally has an idea of the end goal or outcome before he starts. When innovators get stuck with a problem or its solution, and struggle to move it forward, they would increasingly shift gears - use different tools, etc - to shift perspective.

A number of participants report an extensive array of creative thinking tools that they use. Tools are an easy way to start. They highlight some of the principles at play, and help with quick perspective formation. Putting the problem into a similar context that have been solved before helps with context and to define the problem. Innovators may equally be cognizant of their or others' perspectives, so that they can find a way to bring them out of it, in order to challenge it. They look for ways to break familiarity with, preconceptions of, and even prejudice towards a problem. The idea is to get rid of mental filters, and to return to a sense of cognitive innocence or neutrality. The innovator may also work in on the language associated with a problem or solution - changing the language changes the problem or solution definition. The innovator may be able to intuitively provide a different perspective - he may draw on his intuition to give or shift perspective. They frequently shift perspective in the sense of zooming in and out of the problem. The knowledge and experience they have built up, can greatly help them with forming perspective. They learn by facing new challenges. 
Another objective is to balance reasoning, thinking, designing and planning with prototyping to equally introduce the practical aspects. Prototyping is also useful when the innovator wishes to further understand or develop the problem or solution. Failure is seen as constructive, and providing direction. As simple a prototype as possible is built. Learning occurs through practice.

Because it is not possible to have the full experience or perception through cognition only, innovators like to immerse themselves in the problem and its solution. Phenomena must also be perceived through the senses to be really understood. Interaction with a problem helps to better understand it. Involving the senses can invoke emotions with the innovator, that can further contribute to creativity.

Play is also seen as a very prominent stimulant of creativity and creative thought. Play allows the person to be a kid again. It allows free reigns, there are not stereotypes, preconceptions, or boundaries. It can also be seen as a way or method to take the person out of his natural environment - out of what is known or familiar to him, and his routines and thought patterns.

The role of the imagination during creativity is seen to be mostly visualize, model and simulate solutions and ideas. Visualizing the solution helps to demarcate its functionality, properties, attributes, and aesthetics, particularly its physical properties. It helps to articulate the solution or problem. The ability to visualize an idea or solution helps to determine whether it is practical and feasible. The innovator can typically see - visualize - the complete product before it is built. Imagination as simulations and visualizations of scenarios and events, may also be used to better understand problems, helping the innovator to consider things like cause and effect, and social contexts.

Intuition is largely seen to be based on experience. It comes from making mistakes, learning from it, and developing experience from it. When a problem is known or partly known, it is not too difficult for the brain to come up with its solution. By being curious or interested in a problem and its solution, the innovator is essentially involves his sub-conscious. The brain attempts to sub-consciously solve problems, and the innovator allows his mind room to sub-consciously work on the problem. Intuition may play a key role in advancing problems in their initial stage. Often solutions to problems have an intuitive crumb-trial. Even if the innovator does not know the solution, he only needs to know how to advance and work towards a solution. In this sense, the intuition and sub-conscious, and in turn perspective switching, play an important role. Perspective switching, the intuition and the sub-conscious may form a synergy: perspective switching and the intuition may provide the sub-conscious with enough to in turn provide additional content to work with.

The innovator develops a sense of problems, solutions, and contexts. His intuition may guide him towards a solution. His intuition may offer him a glimpse, sense or feeling of an idea or possible solution to a problem, and perhaps the best way to move forward. The innovator may also focus on aspects of an idea or solution that essentially bother him, and may pay attention to it and keep on exploring, until he has no more questions about the problem or solution, or until he can shake off the sense of discomfort, unease, or concern. The sub-conscious may equally realize or perceive problems before the conscious does. Most innovators do not find it difficult to draw from their intuition and sub-conscious, and actively engage their intuition and subconscious to come up with ideas and solutions. They may deliberately or unexpectedly trigger their subconscious. Innovators may rely on their intuition to come up with solutions and ideas, and may indeed source ideas and solutions through their intuition, but ultimately commit to a much more methodological and structured approach to working on ideas and solutions. Ideas and solutions emanating from the intuition are still subjected to objective and systematic evaluation. The fact that aha moments come at random times, and that it is not always possible to explain the steps to a solution, demonstrates the involvement of the subconscious. Intuition may not always be regarded as practical, and thus valued. It may be seen as useless, particularly when the link to creativity is not made. The innovator may also practice and learn to utilize his intuition.

There are 2 divergent views as to what extent it is necessary to define the problem beforehand. Some wish to exactly understand and define the problem. Others see it as only necessary or important to loosely or informally define the problem. Both positions were outlined in detail. Generally, the overall objective is to understand the problem as much and in as much depth as possible.

With regards to utilizing past solutions, there are again 2 opposing views. Some feel that preceding work and past solutions are useful, others feel they are restrictive. Both positions were outlined in detail. An underlying argument is that it would be impossible to separate the innovator from his past knowledge and experience. By implication, this has positive and negative consequences. In a way, there may be differences between past solutions, and past catalysts of solutions - creative moments of the past, and how they were realized. This may help the innovator to be creative again, and to again engage, release or emit creativity. The innovator does not have to be original in method, to be still original in the solution.

The overall view is that there are multiple - or even infinite - solutions, and the innovator generally 
has to choose the best one. The innovator may fall in the trap of running with the first workable solution, without considering and evaluating alternative solutions, to choose the best solution among several candidate solutions. The process of finding the best solution frequently involves trade-offs and is commonly not an easy task.

Interestingly, the innovators interviewed did not readily change their learning style during the creative and innovative process. They stuck to the method and process they knew to approach, interrogate, unpack, and work problems.

Whether the innovator prefer to work in a team, or on his own, depends much on his personality, and the stage of his idea or solution. Some innovators can be very creative either by themselves, or in groups. Some innovators see their creative space as very personal. There are aspects of creative work that the innovator best should do on his own, and there are aspects where he should best involve others. The innovator generally wants someone that will look at the problem or solution differently, so that he and his thinking is challenged by this, and so that his thinking can develop from this. The benefits and complications of groups were outlined.

Working on their own, innovators are generally able to work much faster, and enjoy greater freedom to change direction and consider alternatives. They are less rushed and under pressure. Innovators tend to prefer to do a lot of work beforehand, before articulating and presenting an idea to others. The wrong feedback can discourage, distract and confuse the innovator, and he must be able to manage and assess the input. The background, training, experience, commitment, interest, emotional state and vantage point of those providing input or feedback - contributing - play a huge role. Sometimes the other party may not understand the problem or solution at all, or they may not share the vision of the innovator. Sometimes the problem or solution must be experienced, rather than explained, to be fully appreciated, and the other party may lack the appropriate knowledge to be a true critic. The intricacies of the innovators working on his own were also discussed. An important issue, both in teams and the innovator as individual approaching others, is the level of freedom the innovator is allowed or can expect when approaching others for input and feedback. An interesting question, related to the proposition that SMEs may or may not be more innovative than large enterprises, is whether innovators working on their own are more innovative or effective than teams.

A number of factors that impact the success of the innovator in the market were noted: 1) unique value proposition, 2) business sense or acumen, 3) understanding or knowledge of the market, 4) disrupting and overcoming existing market structures, 5) understanding competition, 6) rendering existing products or solutions irrelevant, 7) commitment, dedication, determination, consistency, time, and effort, 8) understanding and involving customers, 9) shaping market opinions, 10) effective or accurate pitching and marketing, 11) being on time and opportunistic, 12) not being short-sighted, 13) emotional state, psyche and environment, 14) vision, 15) experience, 16) planning, and 17) implementation. The array of factors questions whether creativity is a predominant factor of innovation success - whether innovation success is predominantly determined by creativity alone. Creativity or lack thereof may then not be the most fundamental problem in entrepreneurship.

\section{References}

Abraham, A., Pieritz, K., Thybusch, K., Rutter, B., Kröger, S., Schweckendiek, J., Stark, R., Windmann, S. and Hermann, C., 2012. Creativity and the brain: uncovering the neural signature of conceptual expansion. Neuropsychologia, 50(8), pp.1906-1917.

Antonietti, A. and Colombo, B., 2011. Mental imagery as a strategy to enhance creativity in children. Imagination, Cognition and Personality, 31(1), pp.63-77.

Baron, R.A., 2007. Behavioral and cognitive factors in entrepreneurship: Entrepreneurs as the active element in new venture creation. Strategic Entrepreneurship Journal, 1(1-2), pp.167-182.

Bonnardel, N. and Marmèche, E., 2004. Evocation processes by novice and expert designers: Towards stimulating analogical thinking. Creativity and Innovation Management, 13(3), pp.176-186.

Couger, J.D., Higgins, L.F. and McIntyre, S.C., 1993. (Un) structured creativity in information systems organizations. Mis Quarterly, pp.375-397.

Dayan, M. and Di Benedetto, C.A., 2011. Team intuition as a continuum construct and new product creativity: The role of environmental turbulence, team experience, and stress. Research Policy, 40(2), pp.276286.

Dietrich, A., 2007. Who's afraid of a cognitive neuroscience of creativity?. Methods, 42(1), pp.22-27.

Duch, W., 2007. Intuition, insight, imagination and creativity. IEEE Computational Intelligence Magazine, 2(3), pp.40-52.

Epstein, S., Pacini, R., Denes-Raj, V. and Heier, H., 1996. Individual differences in intuitive-experiential and analytical-rational thinking styles. Journal of personality and social psychology, 71(2), p.390. 
Folkmann, M.N., 2010. Enabling creativity. Imagination in design processes. In DS 66-2: Proceedings of the 1 st International Conference on Design Creativity (ICDC 2010).

Freshwater, D. and Avis, M., 2004. Analysing interpretation and reinterpreting analysis: exploring the logic of critical reflection. Nursing Philosophy, 5(1), pp.4-11.

Garfield, M.J., Taylor, N.J., Dennis, A.R. and Satzinger, J.W., 2001. Modifying paradigms-Individual differences, creativity techniques, and exposure to ideas in group idea generation. Information Systems Research, 12(3), pp.322-333.

Gassmann, O. and Zeschky, M., 2008. Opening up the solution space: the role of analogical thinking for breakthrough product innovation. Creativity and Innovation Management, 17(2), pp.97-106.

Gentner, D., 2010. Bootstrapping the mind: Analogical processes and symbol systems. Cognitive Science, 34(5), pp.752-775.

George, J.M., 2007. 9 Creativity in Organizations. The academy of management annals, 1(1), pp.439-477.

Hargadon, A.B. and Bechky, B.A., 2006. When collections of creatives become creative collectives: A field study of problem solving at work. Organization Science, 17(4), pp.484-500.

Harkow, R.M., 1996. Increasing Creative Thinking Skills in Second and Third Grade Gifted Students Using Imagery, Computers, and Creative Problem Solving.

Harvey, M. and Novicevic, M.M., 2002. The hypercompetitive global marketplace: the importance of intuition and creativity in expatriate managers. Journal of World Business, 37(2), pp.127-138.

Hender, J.M., Rodgers, T.L., Dean, D.L. and Nunamaker, J.F., 2001, January. Improving group creativity: Brainstorming versus non-brainstorming techniques in a GSS environment. In System Sciences, 2001. Proceedings of the 34th Annual Hawaii International Conference on (pp. 10-pp). IEEE.

Henrich, J., 2014. Rice, psychology, and innovation. Science, 344(6184), pp.593-594.

Heppner, M.J., O'Brien, K.M., Hinkelman, J.M. and Humphrey, C.F., 1994. Shifting the paradigm: The use of creativity in career counseling. Journal of Career Development, 21(2), pp.77-86.

Holyoak, K.J. and Thagard, P., 1997. The analogical mind. American psychologist, 52(1), p.35.

Jee, B.D., Uttal, D.H., Gentner, D., Manduca, C., Shipley, T.F., Tikoff, B., Ormand, C.J. and Sageman, B., 2010. Commentary: Analogical thinking in geoscience education. Journal of Geoscience Education, 58(1), pp.2-13.

Kolb, D.A., Boyatzis, R.E. and Mainemelis, C., 2001. Experiential learning theory: Previous research and new directions. Perspectives on thinking, learning, and cognitive styles, 1(8), pp.227-247.

LeBoutillier, N. and Marks, D.F., 2003. Mental imagery and creativity: A meta-analytic review study. British Journal of Psychology, 94(1), pp.29-44.

Leron, U. and Hazzan, O., 2009. Intuitive vs analytical thinking: four perspectives. Educational Studies in Mathematics, 71(3), pp.263-278.

López-González, M. and Limb, C.J., 2012, January. Musical creativity and the brain. In Cerebrum: the dana forum on brain science (Vol. 2012). Dana Foundation.

Lumpkin, G.T. and Lichtenstein, B.B., 2005. The role of organizational learning in the opportunity-recognition process. Entrepreneurship theory and practice, 29(4), pp.451-472.

Mainemelis, C., Boyatzis, R.E. and Kolb, D.A., 2002. Learning styles and adaptive flexibility: Testing experiential learning theory. Management learning, 33(1), pp.5-33.

McFadzean, E., 1998a. The creativity continuum: Towards a classification of creative problem solving techniques. Creativity and Innovation Management, 7(3), pp.131-139.

McFadzean, E., 1998b. Enhancing creative thinking within organisations. Management Decision, 36(5), pp.309-315.

McFadzean, E., 1999. Creativity in MS/OR: choosing the appropriate technique. Interfaces, 29(5), pp.110122.

Mihov, K.M., Denzler, M. and Förster, J., 2010. Hemispheric specialization and creative thinking: A metaanalytic review of lateralization of creativity. Brain and Cognition, 72(3), pp.442-448.

Nolan, V., 2003. Whatever happened to synectics?. Creativity and Innovation Management, 12(1), pp.24-27.

Nuangchalerm, P. and Thammasena, B., 2009. Cognitive Development, Analytical Thinking, and Learning Satisfaction of Second Grade Students learned through Inquiry-based Learning. Asian social science, $5(10)$, p.82.

Pelaprat, E. and Cole, M., 2011. "Minding the gap": Imagination, creativity and human cognition. Integrative Psychological and Behavioral Science, 45(4), pp.397-418.

Raidl, M.H. and Lubart, T.I., 2001. An empirical study of intuition and creativity. Imagination, Cognition and Personality, 20(3), pp.217-230.

Richert, R.A., Whitehouse, H. and Stewart, E., 2005. Memory and analogical thinking in high-arousal rituals. 
Mind and religion: Psychological and cognitive foundations of religiosity, pp.127-145.

Robbins, J.K., 2011. Problem solving, reasoning, and analytical thinking in a classroom environment. The Behavior Analyst Today, 12(1), p.41.

Rusou, Z., Zakay, D. and Usher, M., 2013. Pitting intuitive and analytical thinking against each other: The case of transitivity. Psychonomic bulletin \& review, 20(3), pp.608-614.

Sams, L., 2003. How to teach grammar, analytical thinking, and writing: A method that works. The English Journal, 92(3), pp.57-65.

Sawyer, R.K., 2000. Improvisation and the creative process: Dewey, Collingwood, and the aesthetics of spontaneity. The journal of aesthetics and art criticism, 58(2), pp.149-161.

Shepard, R.N., 1978. Externalization of mental images and the act of creation. Visual learning, thinking, and communication, pp.133-189.

Siegler, R.S., 2006. Microgenetic analyses of learning. Handbook of child psychology.

Sternberg, R.J., 2006. The nature of creativity. Creativity research journal, 18(1), pp.87-98.

Suler, J.R., 1980. Primary process thinking and creativity. Psychological bulletin, 88(1), p.144.

Sutton, R.I. and Hargadon, A., 1996. Brainstorming groups in context: Effectiveness in a product design firm. Administrative Science Quarterly, pp.685-718.

Swann, P. and Birke, D., 2005. How do creativity and design enhance business performance? A framework for interpreting the evidence. DTI Strategy Unit 'Think Piece.

Titus, P.A., 2000. Marketing and the creative problem-solving process. Journal of Marketing Education, 22(3), pp.225-235.

Torrance, E.P., 1993. Understanding creativity: Where to start?. Psychological Inquiry, 4(3), pp.232-234.

von der Malsburg, C., 1997. The coherence definition of consciousness. In Cognition, computation and consciousness (pp. 193-204). Oxford University Press.

Vygotsky, L.S., 1991. Imagination and creativity in the adolescent. Soviet Psychology, 29(1), pp.73-88.

Ward, T.B., 2004. Cognition, creativity, and entrepreneurship. Journal of business venturing, 19(2), pp.173188.

Webster, P.R., 2002. Creative thinking in music: Advancing a model. creativity and music education. Toronto: Canadian Music Educators' Association.

Zeki, S., 2001. Artistic creativity and the brain. Science, 293(5527), pp.51-52. 